\title{
Supporting Information for: \\ Comprehensive evaluation of a quantitative shotgun lipidomics platform for mammalian sample analysis on a high-resolution mass spectrometer
}

\author{
Inger Ødum Nielsen ${ }^{1}$, André Vidas Olsen², Jano Dicroce Giacobini', Elena Papaleo ${ }^{2,3}$, Klaus
} Kaae Andersen ${ }^{4}$, Marja Jäättelä ${ }^{1,5}$, Kenji Maeda ${ }^{1}$, Mesut Bilgin $^{1 *}$

${ }^{1}$ Unit for Cell Death and Metabolism, Center for Autophagy, Recycling and Disease, Danish Cancer Society Research Center, Copenhagen DK-2100, Denmark.

${ }^{2}$ Computational Biology Laboratory, Center for Autophagy, Recycling and Disease, Danish Cancer Society Research Center, DK-2100, Denmark.

${ }^{3}$ Translational Disease Systems Biology, Novo Nordisk Foundation Center for Protein Research University of Copenhagen, Copenhagen DK-2200, Denmark.

${ }^{4}$ Unit for Statistics and Epidemiology, Danish Cancer Society Research Center, Copenhagen DK2100, Denmark.

${ }^{5}$ Department of Cellular and Molecular Medicine, University of Copenhagen, Copenhagen DK-2200, Denmark

*corresponding author: mesutb@ cancer.dk

KEYWORDS: shotgun lipidomics, evaluation, mammalian lipidome, high-resolution mass spectrometry 
Supporting Table 1. Operational ionization modes, $\mathrm{m} / \mathrm{z}$, ranges, and normalized collision energies (nCE) applied in the FT MS and FT MS/MS spectra acquisition.

Supporting Table 2. Overview on the titration experiments with pairs of synthetic lipid standards belonging to 26 lipid classes and the determined dynamic quantification ranges.

Supporting Figure 1. Titrations with 26 pairs of synthetic lipids to determine dynamic quantification ranges for 38 lipid classes.

Supporting Figure 2. Dynamic quantification ranges of lipids in MEF Src samples presented in $\mathrm{pmol} / \mathrm{cell}$.

Supporting Figure 3. Titrations with pairs of synthetic CE and SM species to determine dynamic quantification ranges in murine plasma.

Supporting Figure 4. Determination of optimal collision energies for 27 synthetic lipid species belonging to 20 classes in the positive ionization mode in our lipidomics platform.

Supporting Figure 5. Determination of optimal collision energies for 28 synthetic lipid species belonging to 27 classes in the negative ionization mode in our lipidomics platform.

Supporting Figure 6. Determination of optimal working amount for murine brain samples presented in $\mathrm{pmol} / \mu \mathrm{g}$ total protein.

Supporting Figure 7. \%Cv values calculated for the quantities of lipid species determined using increasing brain sample amount.

Supporting Figure 8. Determination of optimal working amount for cultured MCF7 cell samples.

Supporting Figure 9. Determination of optimal working amount for murine plasma samples.

Supporting Figure 10. Determination of optimal working amount for cultured MCF7 cell samples presented in $\mathrm{pmol} / \mathrm{cell}$. 
Supporting Figure 11. Determination of optimal working amount for murine plasma samples as $\mathrm{pmol} / \mu \mathrm{L}$ plasma.

Supporting Figure 12. Reproducibility of quantitative lipidomics data acquired with our platform.

Supporting Figure 13. Effects of serum conditions on the lipidome of cultured MCF7 cells.

Supporting Figure 14. Effects of serum conditions on the lipidome of cultured MCF7 cells.

Supporting Figure 15. Effects of confluency on the lipidome of cultured HeLa cells.

Supporting Figure 16. Effects of confluency on the lipidome of cultured HeLa cells.

Supporting Figure 17. Effects of detachment methods on the lipidome of cultured HeLa cells.

Supporting Figure 18. Effects of detachment methods on the lipidome of cultured HeLa cells.

Supporting Figure 19. Effects of transfection reagents on the lipidome of cultured MCF7 cells.

Supporting Figure 20. Effects of transfection reagents on the lipidome of cultured MCF7 cells.

Supporting Figure 21. Quantified levels of BMP and PG species in MCF7 cells, based on LC-MS data. 
Supporting Table 1. Operational ionization modes, $\mathrm{m} / \mathrm{z}$, ranges, and normalized collision energies (nCE) applied in the FT MS and FT MS/MS spectra acquisition of the targeted 38 lipid classes. The "mass-tag" strategy is optionally used in the lipidomics platform and monitors LCB after chemical methylation with $\mathrm{CD}_{3} \mathrm{I}$.

\begin{tabular}{l|ccr}
\hline \hline POS ionization mode & FT-MS, $\boldsymbol{m} / \boldsymbol{z}$ & FT-MS/MS, $\boldsymbol{m} / \boldsymbol{z}$ & Lipid classes \\
\hline Low range & $375-730$ & $\begin{array}{c}375.3-728.6 \\
(20 \& 27.5 \% \mathrm{nCE})\end{array}$ & LSM, Cer, DAG, LPC, LPC O- \\
\cline { 2 - 4 } High range & & $574.4-1201.8$ & CE, HexCer, diHexCer, triHexCer, PC, \\
& $550-1200$ & $(20 \& 30 \% \mathrm{nCE})$ & PC O-, SM, TAG \\
Selected ion monitoring & $402-410$ & $404.39 \& 408.41$ & Chol \\
\cline { 2 - 4 } (SIM) & & $(12.5 \% \mathrm{nCE})$ & $\left(\mathrm{CD}_{3}\right)_{3}$-LCB \\
\hline
\end{tabular}

NEG ionization mode

\begin{tabular}{l|ccr}
\hline Low range & $350-675$ & $\begin{array}{c}350.16-675.4 \\
(22.5 \& 32.5 \% \mathrm{nCE})\end{array}$ & $\begin{array}{r}\text { LCBP, LPA, LPA O- LPE, LPE O-, LPG, } \\
\text { LPG O-, LPI, LPI O-, LPS, LPS O- }\end{array}$ \\
\cline { 2 - 4 } High range & $500-1050$ & $\begin{array}{c}500.3-1050.7 \\
(20 \& 32.5 \% \mathrm{nCE})\end{array}$ & $\begin{array}{r}\text { CerP, PA, PA O-, PE, PE O-, PG, PG O-, } \\
\text { PI, PI O-, PS, PS O-, SHexCer }\end{array}$ \\
\hline \hline
\end{tabular}


Supporting Table 2. Overview on the titration experiments with pairs of synthetic lipid standards belonging to 26 lipid classes and the determined dynamic quantification ranges. The titrant and constant lipid amounts are listed (pmol) together with the determined dynamic quantification ranges spanning from 0.1-9,000 pmol.

\begin{tabular}{|c|c|c|c|c|}
\hline \multicolumn{2}{|c|}{$\begin{array}{c}\text { Titrant lipid ranges } \\
\text { (pmol) }\end{array}$} & \multicolumn{2}{|c|}{$\begin{array}{c}\text { Constant lipids amount } \\
\text { (pmol) }\end{array}$} & \multirow{2}{*}{$\begin{array}{c}\begin{array}{c}\text { Dynamic quantification range } \\
\text { (pmol) }\end{array} \\
2.7-900\end{array}$} \\
\hline CE 15:0-D7 & $0.1-900$ & CE 15:0 & 17.5 & \\
\hline Cer 18:1;2/12:0;0 & $0.1-900$ & Cer 35:1 & 7.7 & $0.3-900$ \\
\hline CerP 18:1;2/12:0;0 & $0.1-900$ & CerP 18:1;2/16:0;0 & 7.3 & $0.5-450$ \\
\hline Chol-D 4 & $1-9000$ & Chol-D7 & 150 & $27-9000$ \\
\hline DAG 12:0/12:0 & $0.1-900$ & DAG 10:0/10:0 & 20.6 & $0.3-900$ \\
\hline diHexCer 18:1;2/12:0;0 & $0.1-900$ & diHexCer 18:1;2/17:0;0 & 7.6 & $0.3-900$ \\
\hline HexCer 18:1;2/12:0;0 & $0.1-900$ & HexCer 18:1;2/17:0;0 & 12.4 & $0.3-900$ \\
\hline$\left(C_{3}\right)_{3}-$ LCB $17: 1 ; 2$ & $0.1-900$ & $\left(\mathrm{CD}_{3}\right)_{3}-\mathrm{LCB} 17: 0 ; 2$ & 56.5 & $0.9-900$ \\
\hline LCBP & $0.1-900$ & LCBP 17:0;2 & 26.6 & $0.9-450$ \\
\hline LPA 17:0 & $0.1-900$ & LPA 13:0 & 70.5 & $0.3-750$ \\
\hline LPC 10:0 & $0.1-900$ & LPC 12:0 & 17.5 & $0.2-900$ \\
\hline LPE 13:0 & $0.1-900$ & LPE $17: 1$ & 6.7 & $0.3-900$ \\
\hline LPG 17:1 & $0.1-900$ & LPG 13:0 & 9.5 & $0.2-900$ \\
\hline LPI 13:0 & $0.1-900$ & LPI 17:1 & 14.2 & $0.3-600$ \\
\hline LPS $17: 1$ & $0.1-900$ & LPS 13:0 & 13.2 & $0.3-900$ \\
\hline LSM 17:1;2 & $0.1-900$ & LSM 18:1;2 & 6.4 & $0.3-600$ \\
\hline PA 12:0/12:0 & $0.1-900$ & PA 17:0/14:1 & 5.2 & $0.1-218.7$ \\
\hline PC 12:0/12:0 & $0.1-900$ & PC 23:0/23:0 & 56 & $0.3-900$ \\
\hline PE 12:0/12:0 (NEG) & $0.1-900$ & PE 10:0/10:0 & 59.75 & $0.2-900$ \\
\hline PE 12:0/12:0 (POS) & $0.1-900$ & PE 10:0/10:0 & 59.75 & $0.2-900$ \\
\hline PG 12:0/12:0 & $0.1-900$ & PG 17:0/14:1 & 1.9 & $0.3-600$ \\
\hline PI 8:0/8:0 & $0.1-900$ & PI 17:0/14:1 & 4.0 & $0.3-600$ \\
\hline PS 12:0/12:0 & $0.1-900$ & PS 17:0/14:1 & 3.5 & $0.3-750$ \\
\hline SHexCer 18:1;2/12:0;0 & $0.1-900$ & SHexCer 18:1;2/17:0;0 & 10.29 & $0.3-900$ \\
\hline SM 18:1;2/12:0;0 & $0.1-900$ & SM 18:1;2/17:0;0 & 9.4 & $0.3-900$ \\
\hline TAG 17:0/17:0/17:0 & $0.1-900$ & $D_{5}$-TAG 15:0/18:1/15:0 & 14.1 & $0.2-900$ \\
\hline triHexCer 18:1;2/17:0;0 & $0.1-900$ & triHexCer 18:1;2/16:0;0 & 33.85 & $24.3-750$ \\
\hline
\end{tabular}



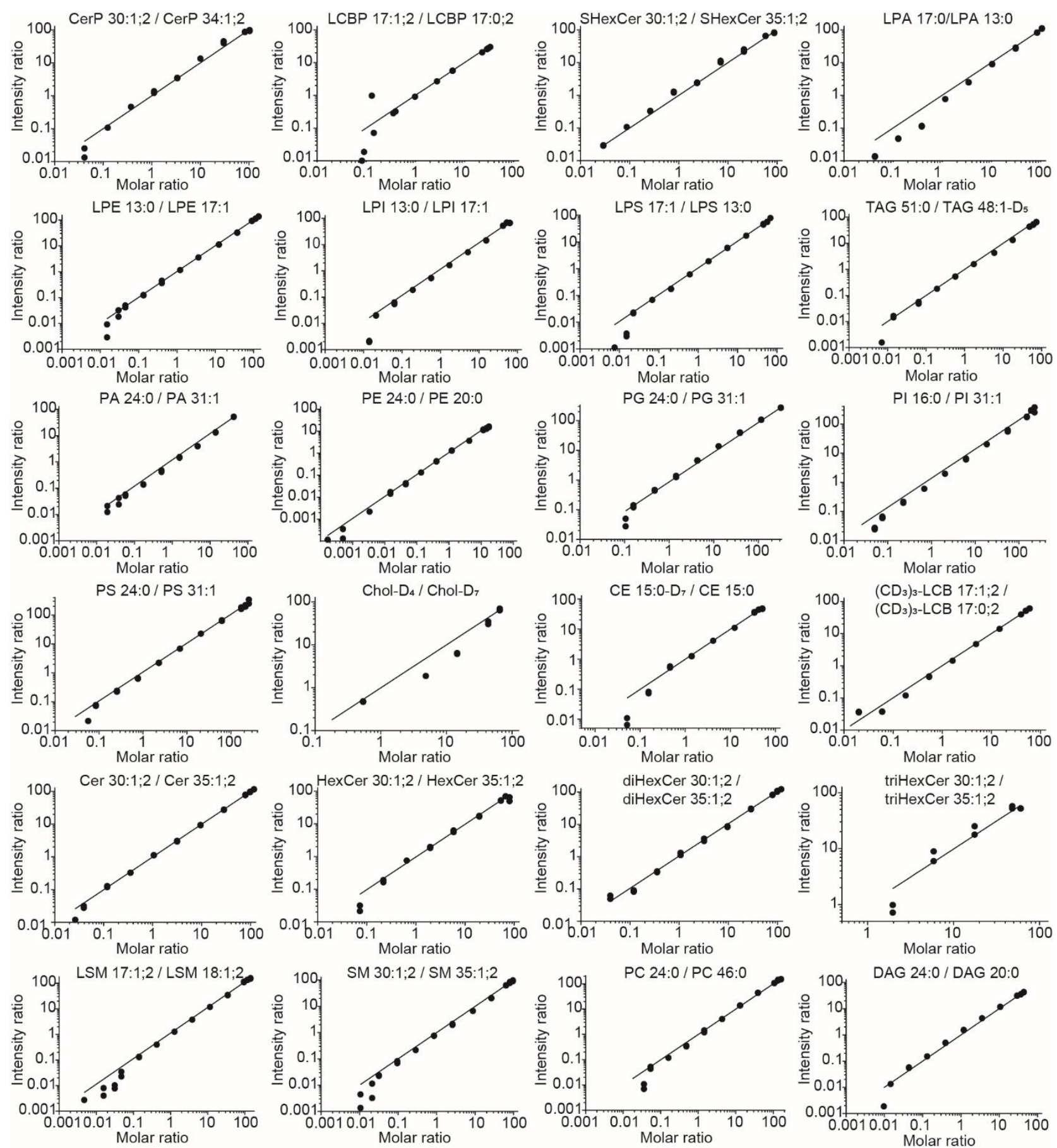

Supporting Figure 1. Titrations with 26 pairs of synthetic lipids to determine dynamic quantification ranges for 38 lipid classes in MEF Src samples. The prepared total lipid extracts were analyzed in the positive and negative ionization mode FT MS using target resolution of 140,000 at $\mathrm{m} / \mathrm{z}$ 200. A synthetic lipid was kept in a constant amount and was titrated with another species of same lipid class (for details see Supporting Table 2). The x-axis shows the molar ratios of the titrant and the constant lipids. The y-axis shows the intensity ratios of the titrant and the constant lipids. The line indicates a linear function $\mathrm{y}=\mathrm{x}$. Depicted values derive from two replicate analyses. 


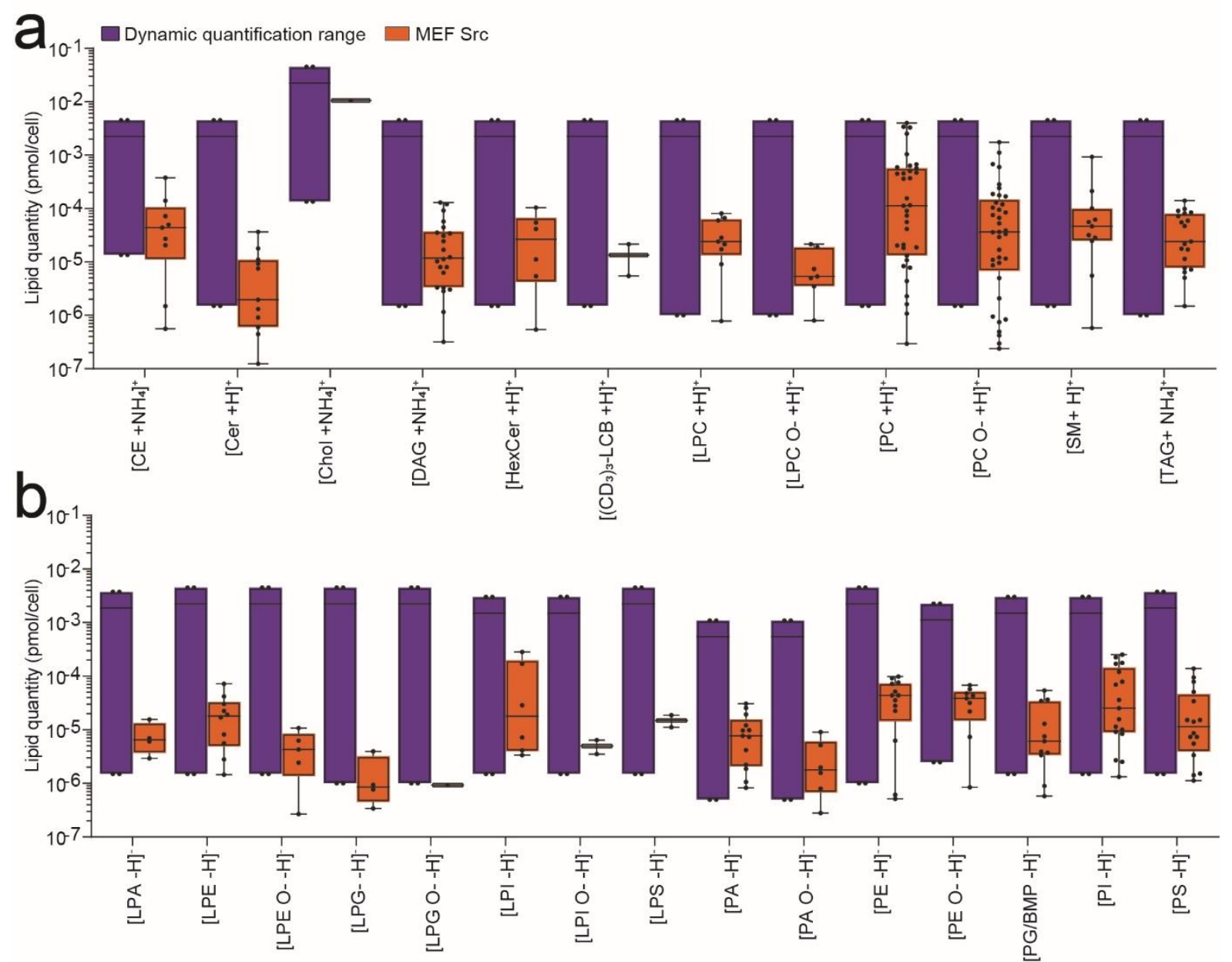

Supporting Figure 2. Dynamic quantification ranges of lipids in MEF Src samples presented in pmol/cell. (a, b) The determined dynamic quantification range of 27 lipid classes analyzed in the positive (a) and negative (b) ionization modes. The box plot shows distributions of determined quantities for species belonging to the 27 lipid classes determined for MEF Src samples. 

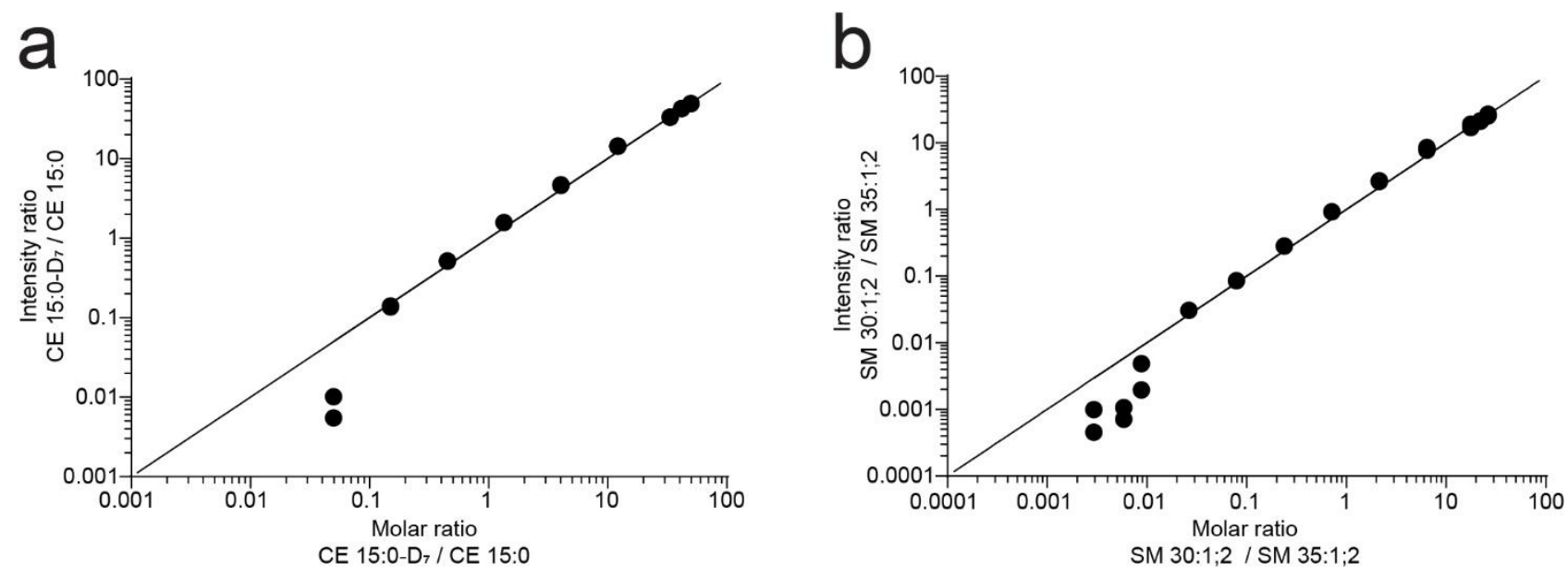

Supporting Figure 3. Titrations with pairs of synthetic CE and SM species to determine dynamic quantification ranges in murine plasma. The prepared total murine plasma lipid extracts were analyzed in the positive ionization mode FT MS using target resolution of 140,000 at $\mathrm{m} / \mathrm{z}$ 200. (a) Titration of 18 pmol CE 15:0 with 0.1-900 pmol CE 15:0-D7 with demonstrating dynamic quantification range spanning from 2.7-900 pmol. The line indicates a linear function $\mathrm{y}=\mathrm{x}$. Depicted values derive from two replicated analyses. (b) Titration of 34 pmol SM 35:1;2 with 0.1-900 pmol SM 30:1;2 demonstrating with dynamic quantification range spanning from $0.9-900$ pmol. The line indicates a linear function $y=$ x. Depicted values derive from two replicated analyses. 
DAG 20:0

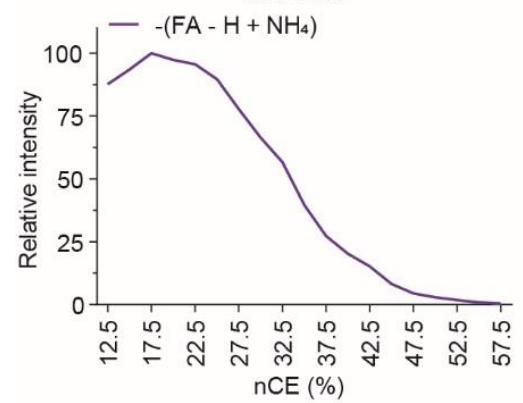

Chol-D 4

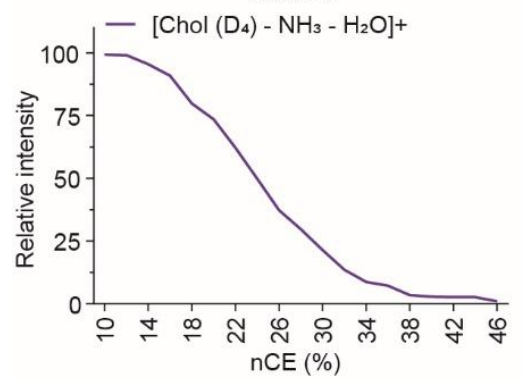

LPE 17:1

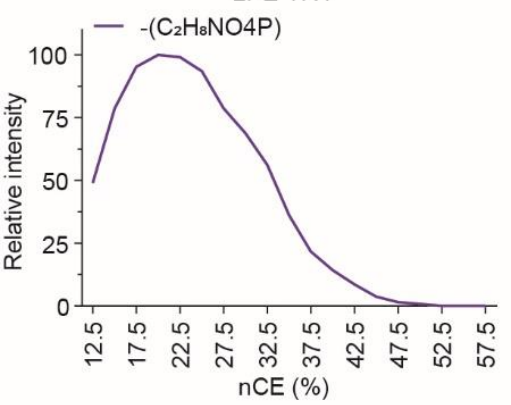

PE 31:1

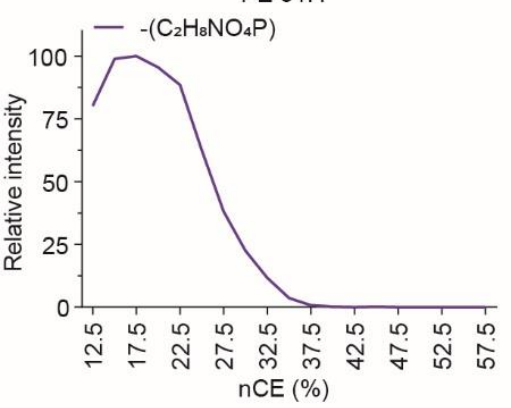

LPC 17:0

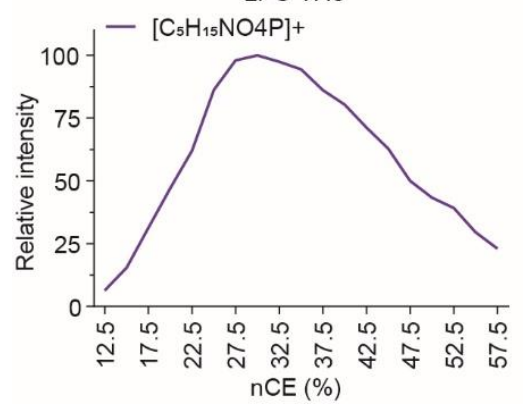

DAG 24:0

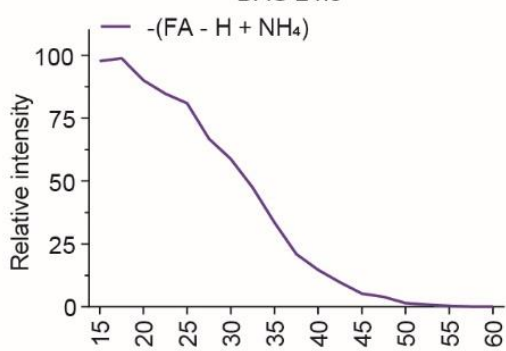

nCE (\%)

CE 15:0-D7

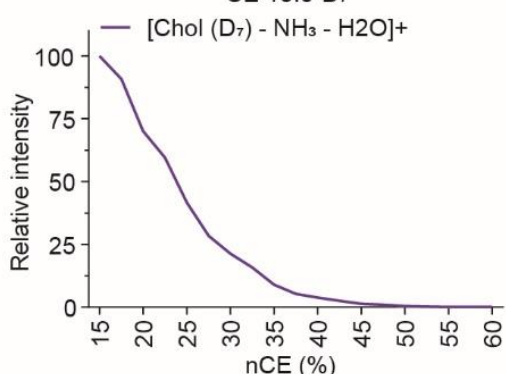

PE 20:0

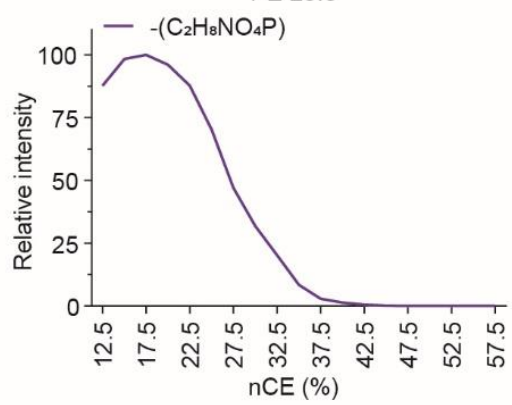

LPC 10:0

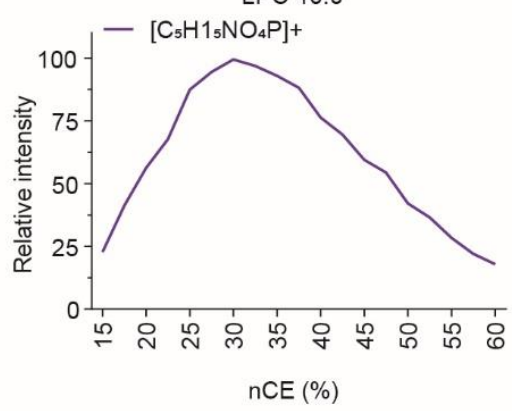

PC 24:0

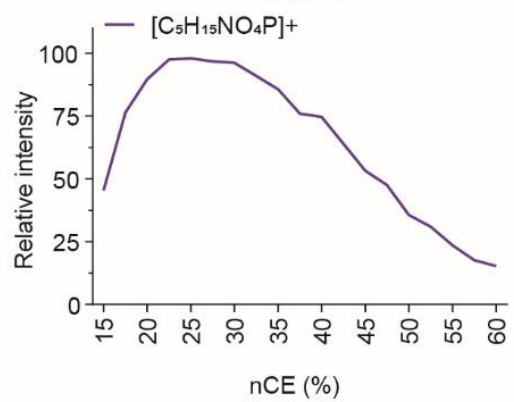

TAG 51:0

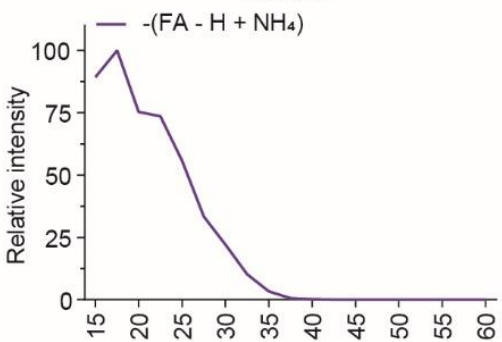

nCE (\%)

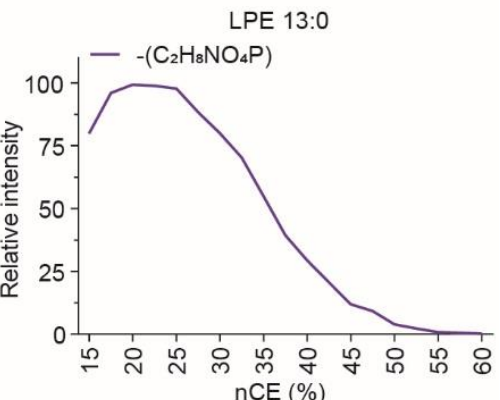

PE 24:0

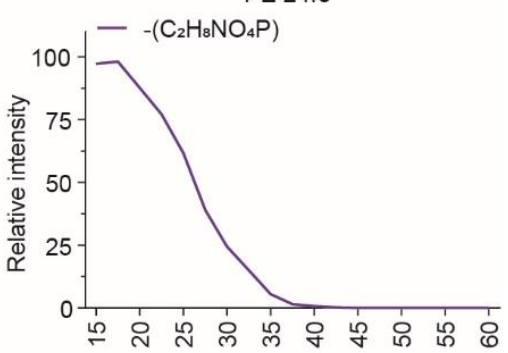

nCE (\%)

LPC 12:0

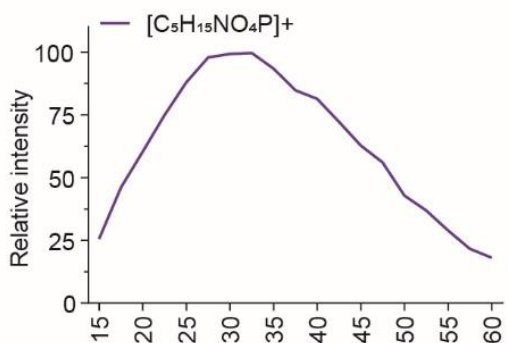

nCE (\%)

PC 36:4

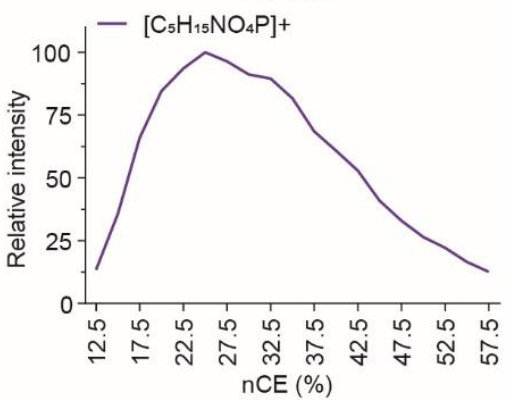




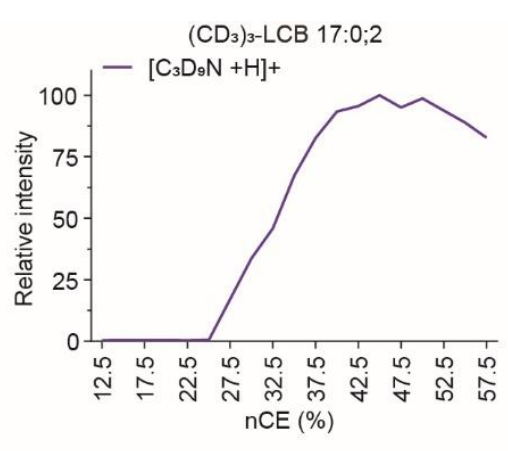

Cer $35: 1 ; 2$

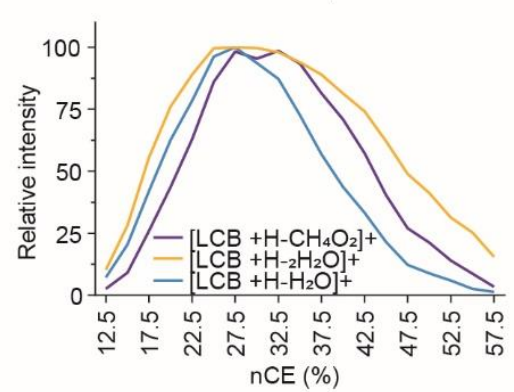

diHexCer 30:1;2

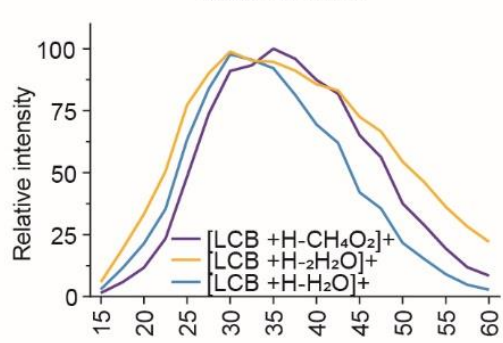

nCE (\%)

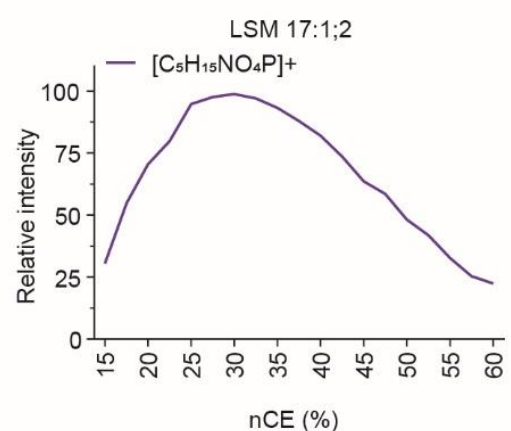

$\left(\mathrm{CD}_{3}\right)_{3}-\mathrm{LCB} 17: 1 ; 2$

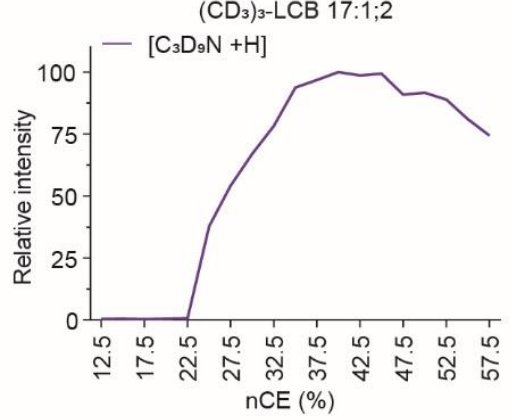

HexCer 30:1;2

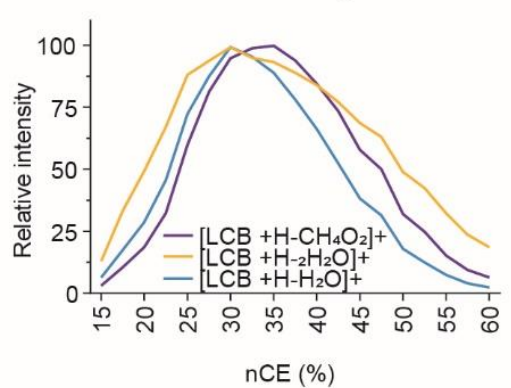

diHexCer 35:1;2
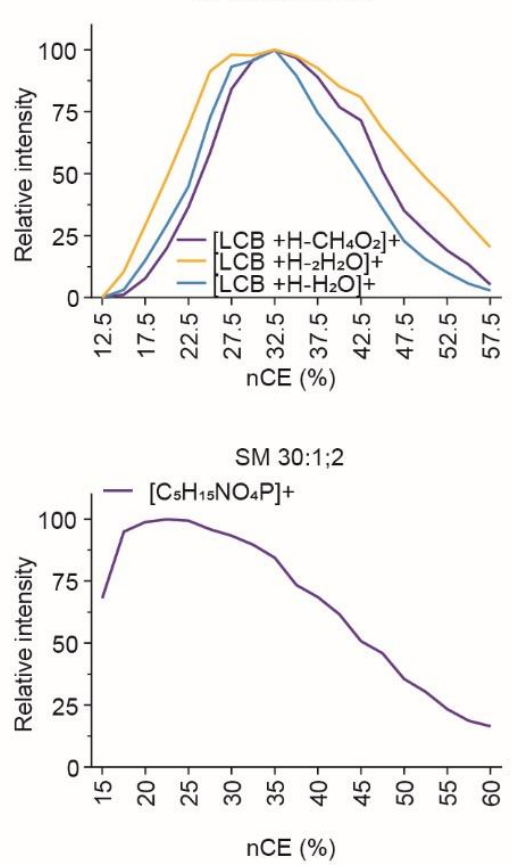

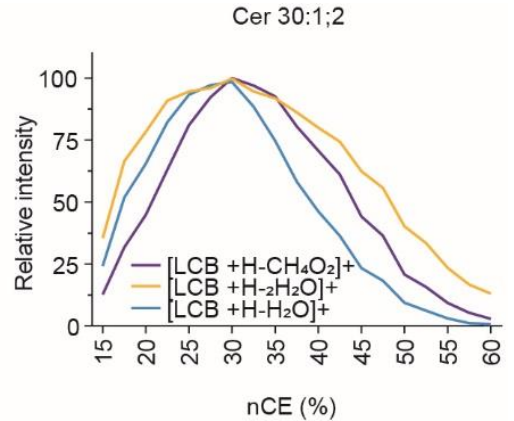

HexCer 35:1;2

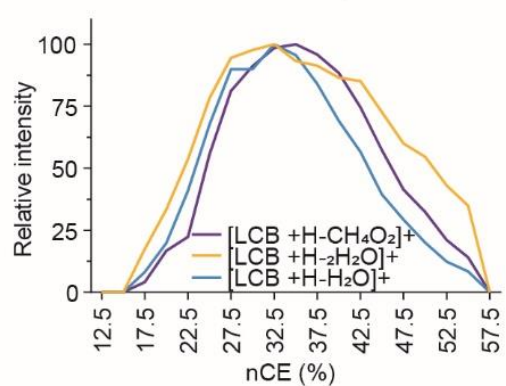

triHexCer 35:1;2
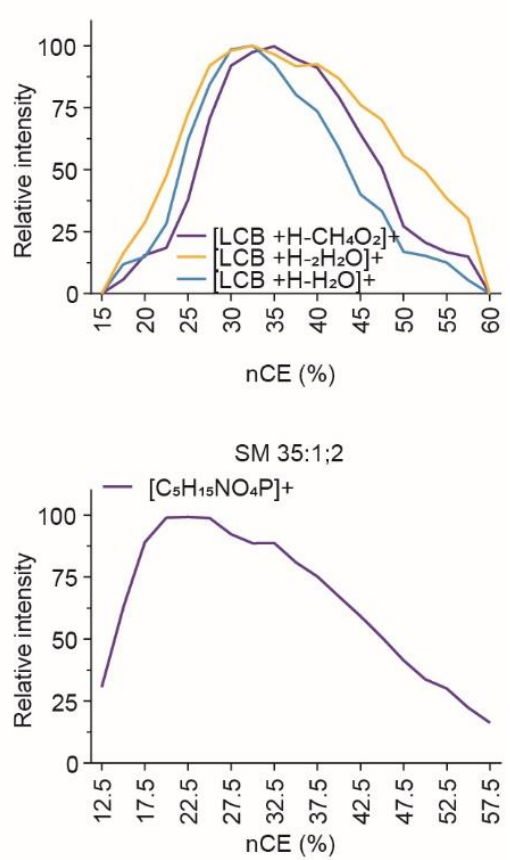

Supporting Figure 4. Determination of optimal collision energies for 27 synthetic lipid species belonging to 20 classes in the positive ionization mode. Plots show the recorded relative intensities of fragment ions as functions of the applied collision energies. 

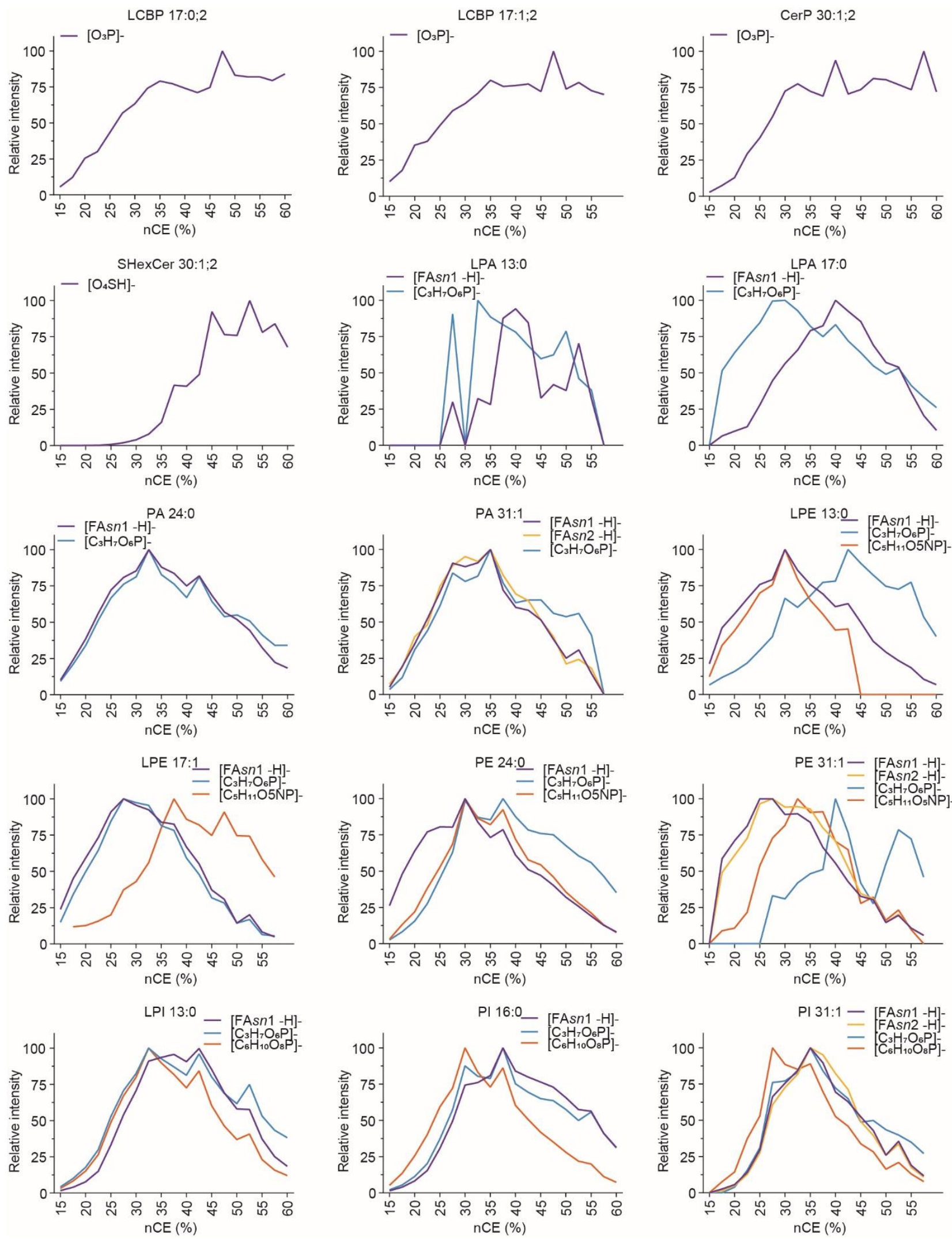

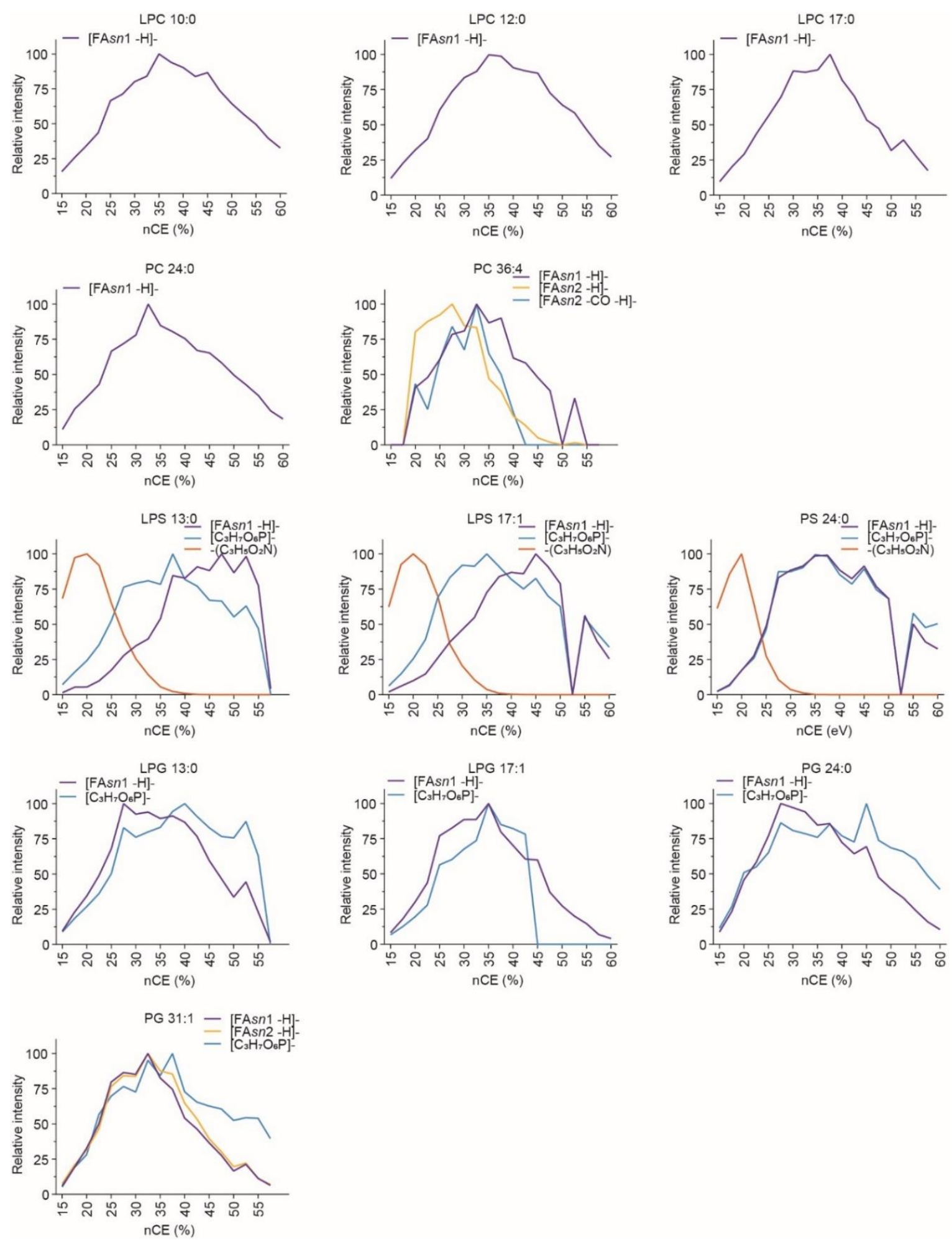

Supporting Figure 5. Determination of optimal collision energies for 28 synthetic lipid species belonging to 27 classes in the negative ionization mode. Plots show the recorded relative intensities of fragment ions as functions of the applied collision energies. For unknown reasons, no intensities were recorded for the fragmentation of LPA 13:0 at nCE 30\%, PC 36:4 at nCE 50\%, LPS 17:1 at nCE 52.5\%, and PS 24:0 at nCE 52.5\%. 

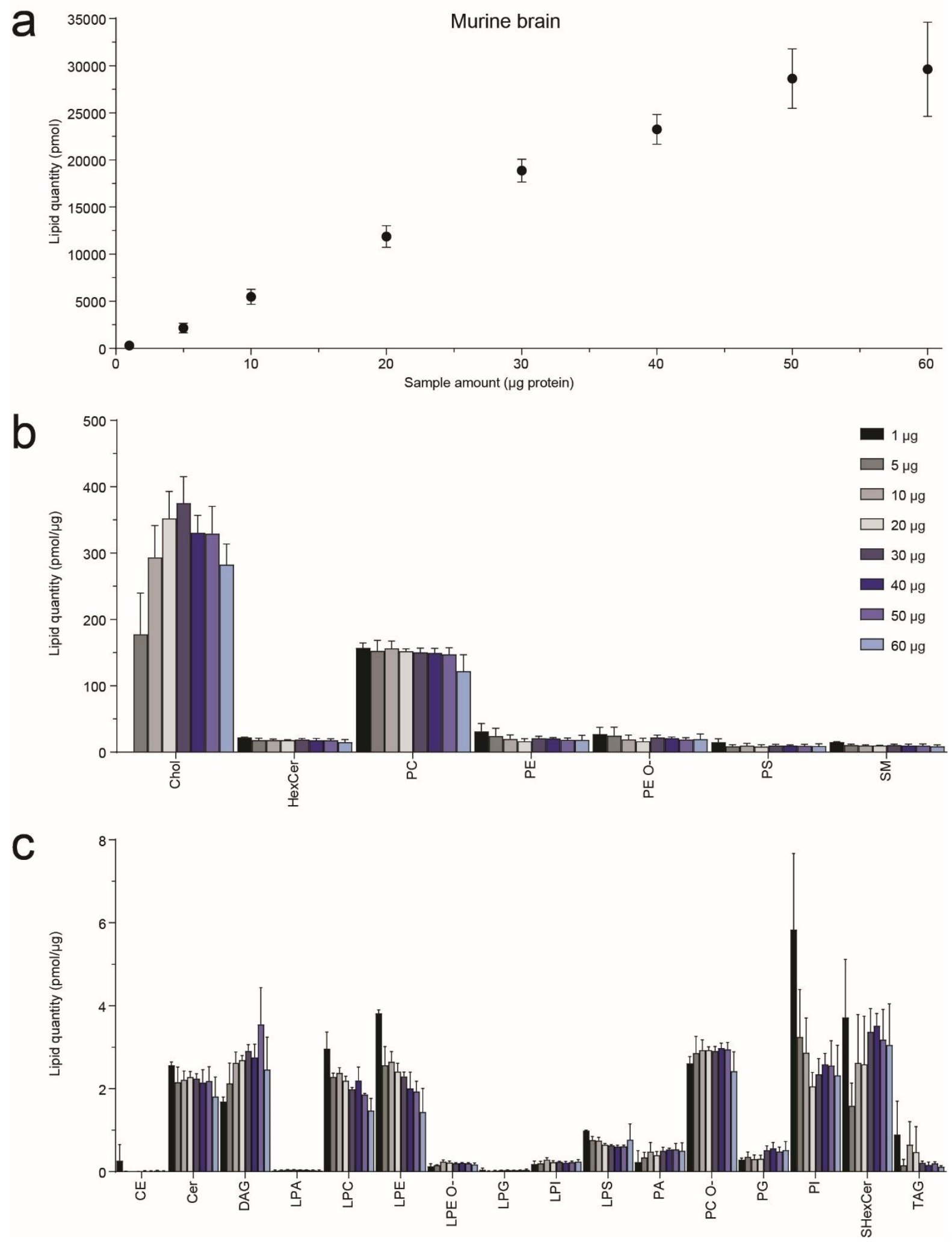

Supporting Figure 6. Determination of optimal working amount for murine brain samples presented in pmol/ $\mu \mathrm{g}$ total protein. (a) Total molar quantities of lipids identified in increasing amount of murine brain homogenate. (b, c) Quantitative profiles of lipid classes determined for the murine brain homogenate using different sample amounts. 

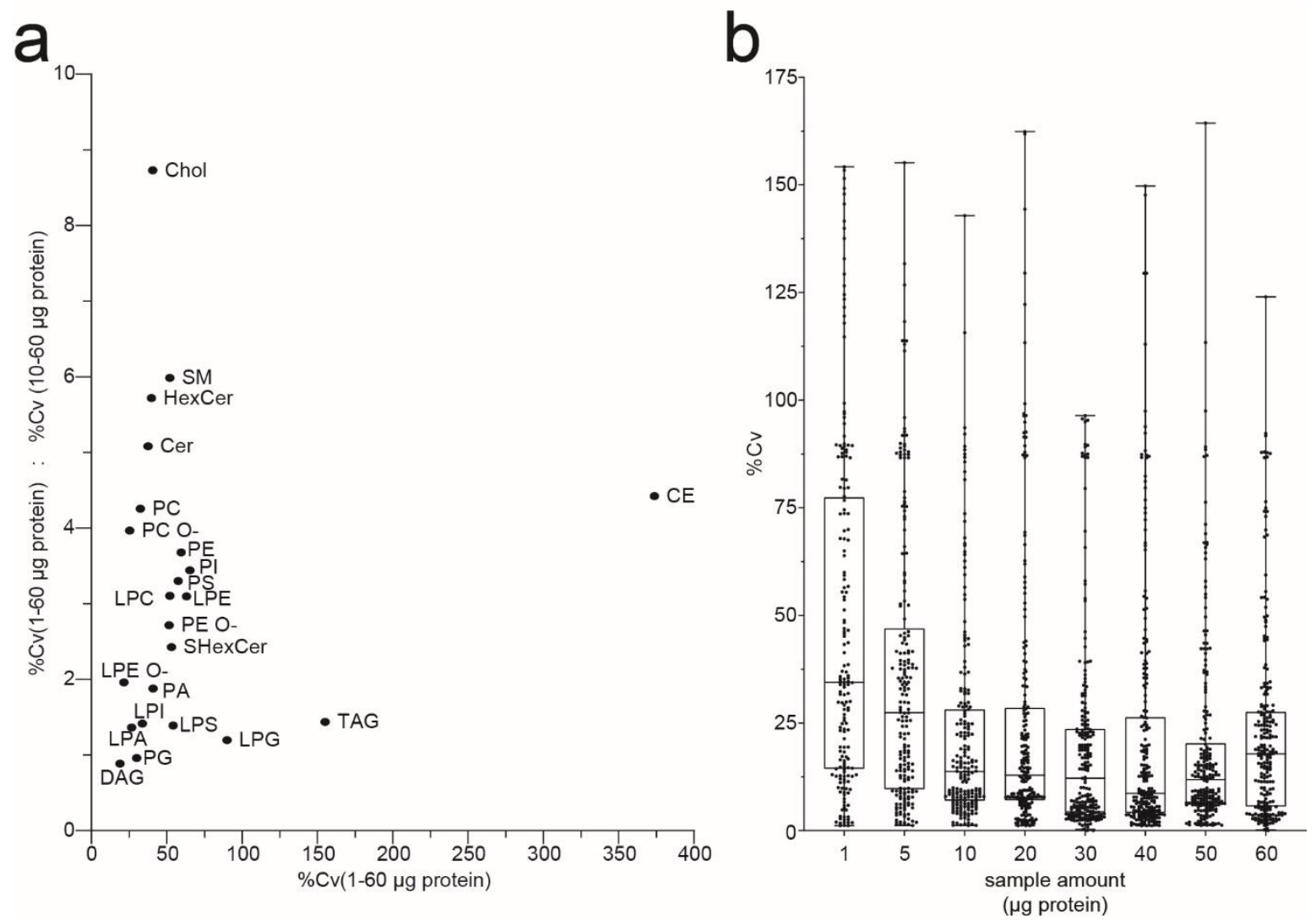

Supporting Figure 7. \% Cv values calculated for the determined quantities of lipid species. (a) $\% \mathrm{Cv}$ values calculated for levels of lipid classes determined with sample amounts of 1-60 $\mu$ g total protein content plotted against corresponding values with $10-60 \mu \mathrm{g}$ total protein content. (b) $\% \mathrm{Cv}$ values calculated for the quantities of lipid species determined with various sample amount of 1-60 $\mu \mathrm{g}$ total protein contents. 

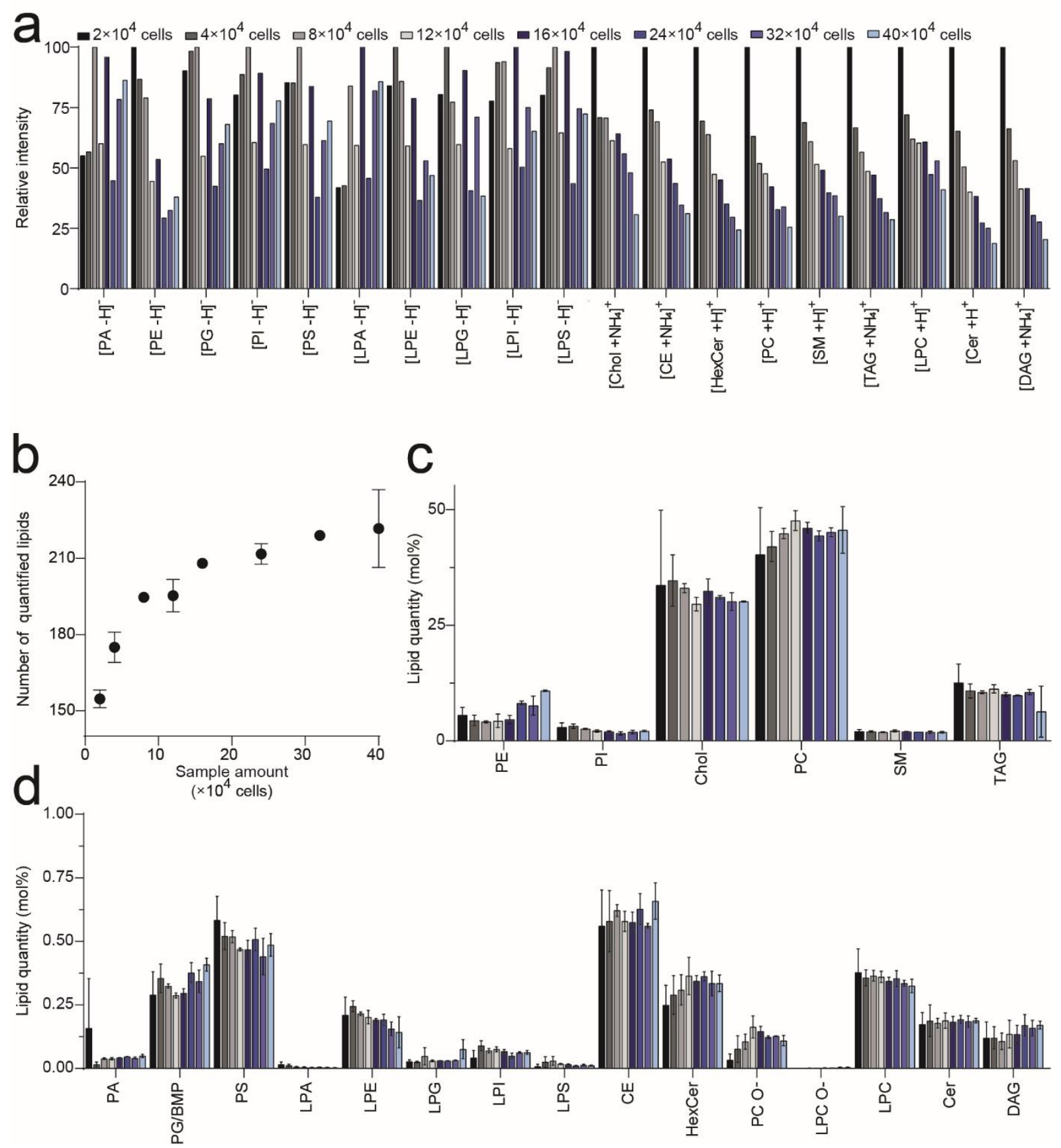

Supporting Figure 8. Determination of optimal working amount for cultured MCF7 cell samples. (a) Relative ion intensities of internal lipid standards measured with FT MS after spiking them into increasing numbers of MCF7 cells. The intensities are shown relative to the highest measured intensity of the standard. (b) Numbers of lipid species identified in MCF7 cells using different sample amounts. (c, d) Quantitative profiles of lipid classes in MCF7 cells determined using different sample amounts. Lipid classes with levels above (c) and below (d) 1 mol\%. 

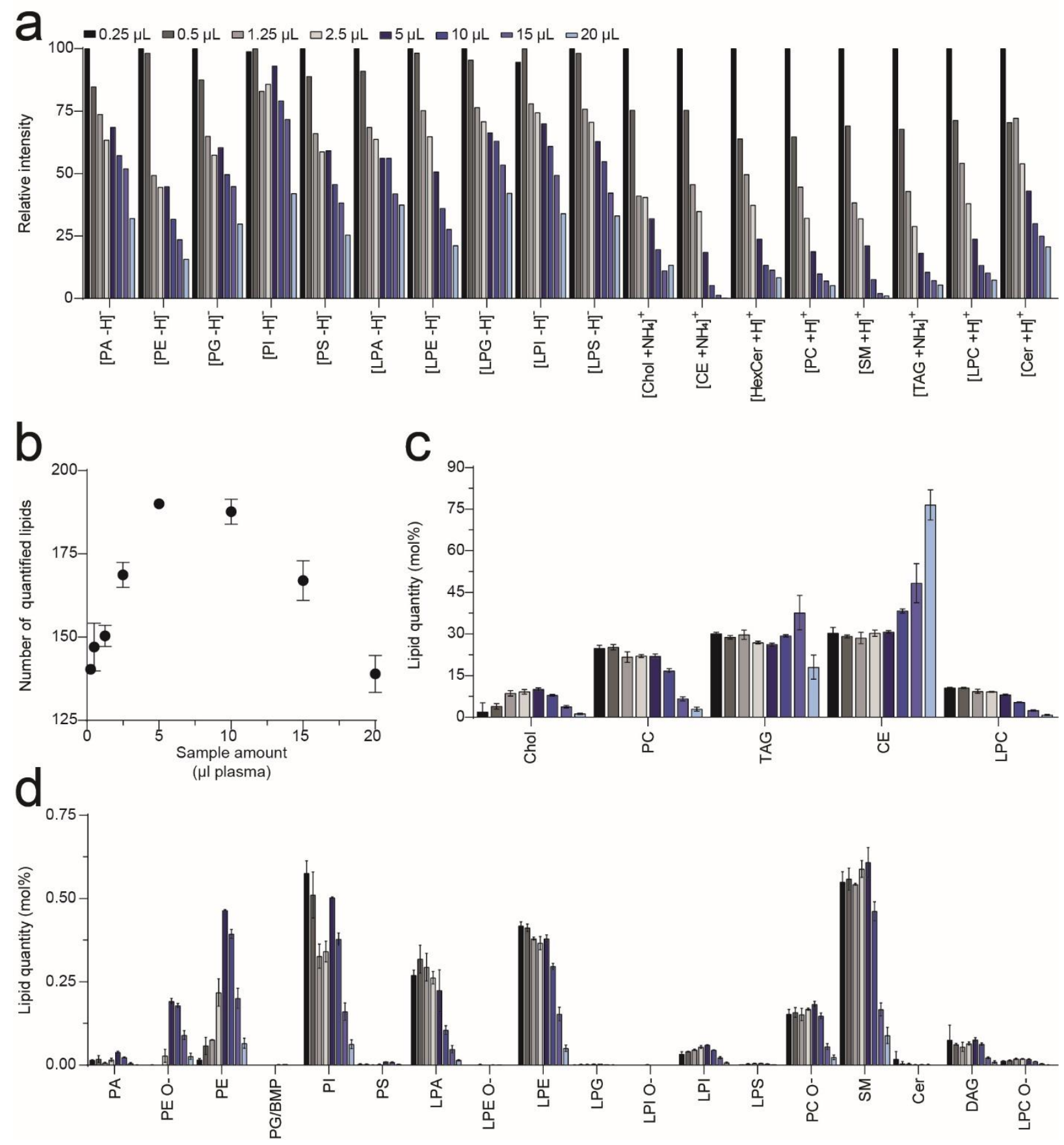

Supporting Figure 9. Determination of optimal working amount of murine plasma for quantitative shotgun lipidomics.

(a) Relative ion intensities of internal lipid standards measured with FT MS after spiking them into increasing volumes of murine plasma. The intensities are shown relative to the highest measured intensity of the standard. (b) Numbers of lipid species identified in murine plasma using different sample volumes. (c, d) Quantitative profiles of lipid classes in murine plasma determined using different sample volumes. Lipid classes with levels above (c) and below (d) $0.75 \mathrm{~mol} \%$. 

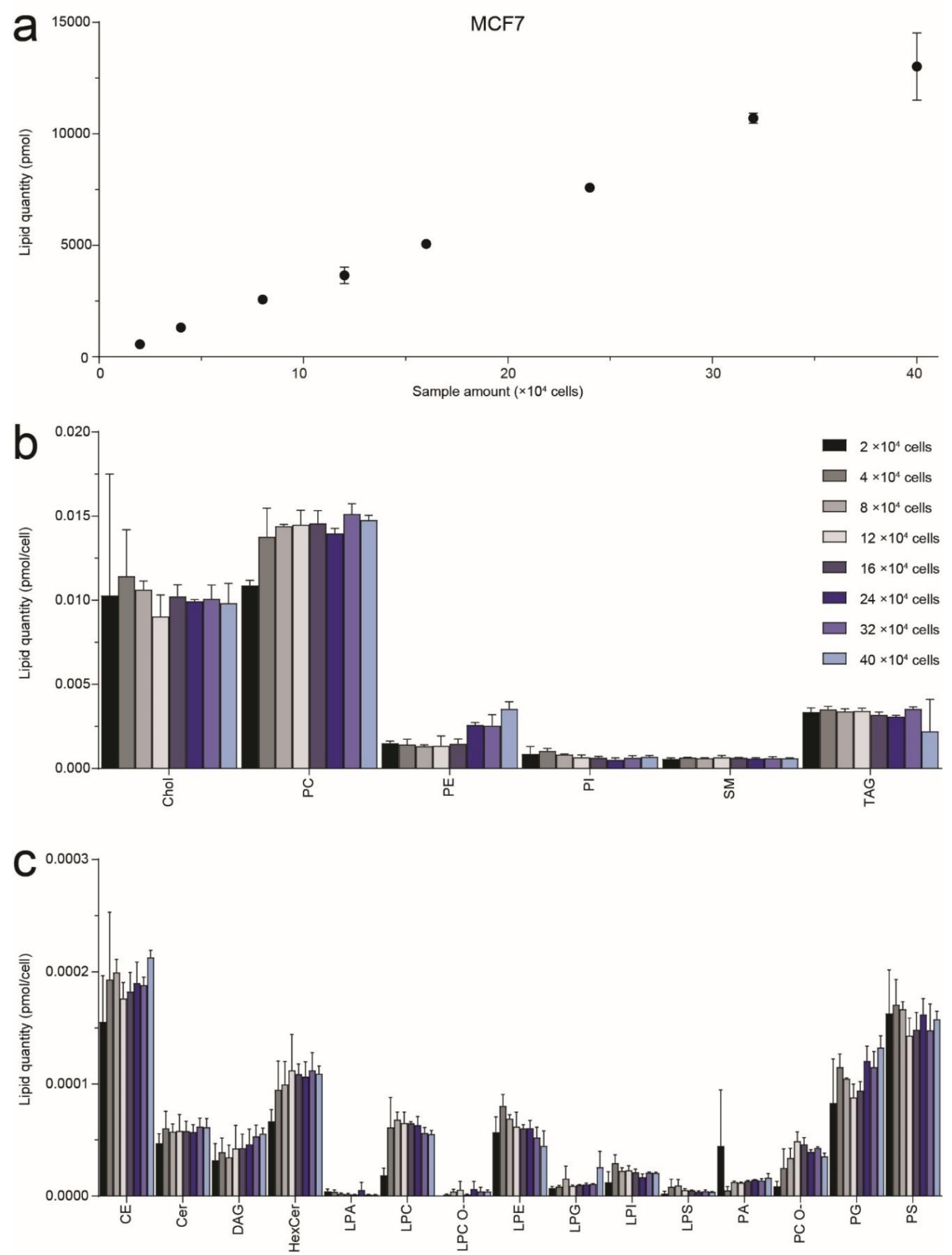

Supporting Figure 10. Determination of optimal working amount for cultured MCF7 cell samples presented in pmol/cell. (a) Total molar quantities of lipids identified in increasing numbers of MCF7 cells. (b, c) Quantitative profiles of lipid classes in MCF7 cells determined using different sample amounts. 

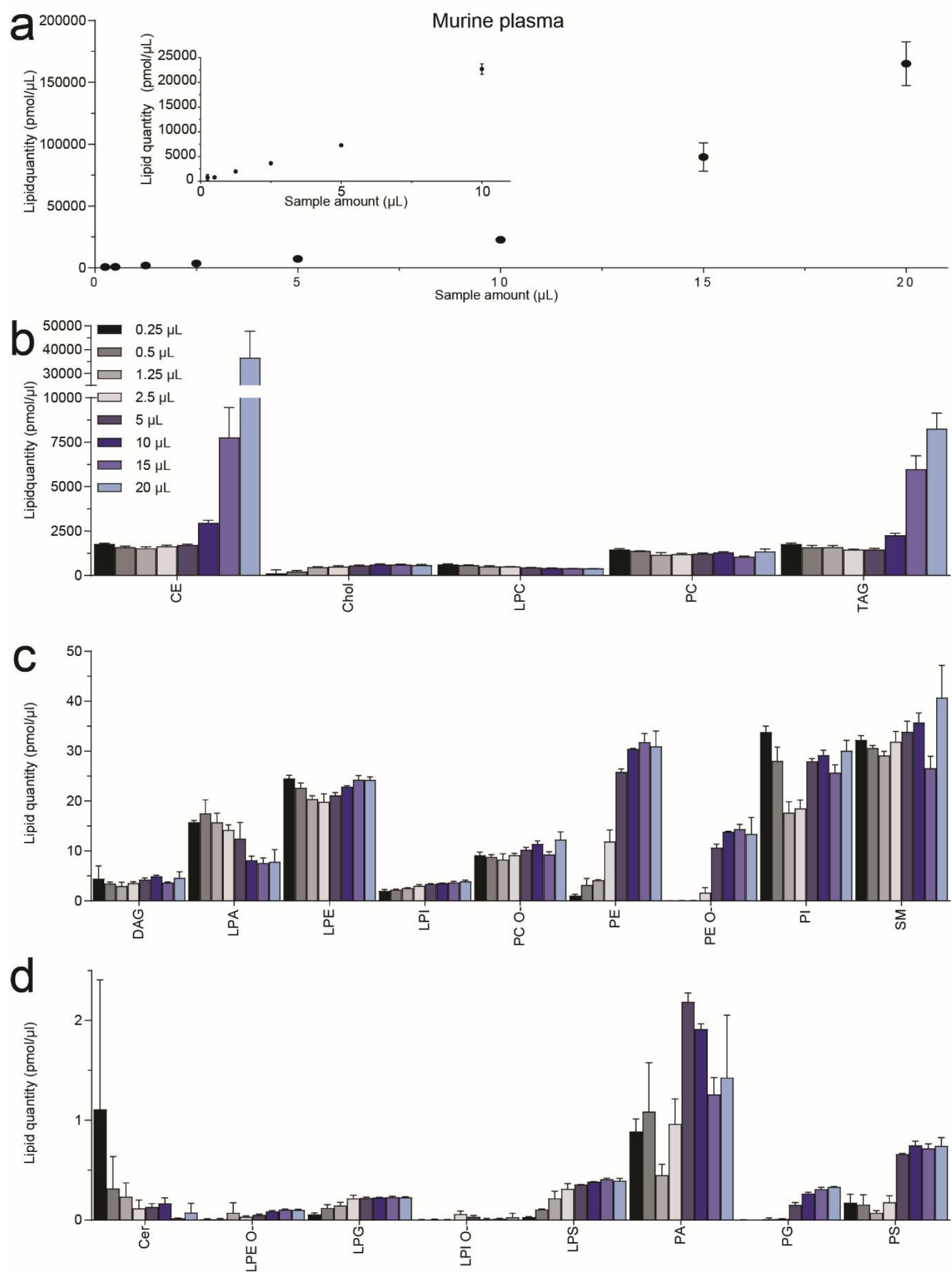

Supporting Figure 11. Determination of optimal working amount for murine plasma samples as pmol/ $\mu \mathrm{L}$ plasma. (a) Total molar quantities of lipids identified in increasing volumes of murine plasma. (b-d) Quantitative profiles of lipid classes in murine plasma determined using different sample volumes. 

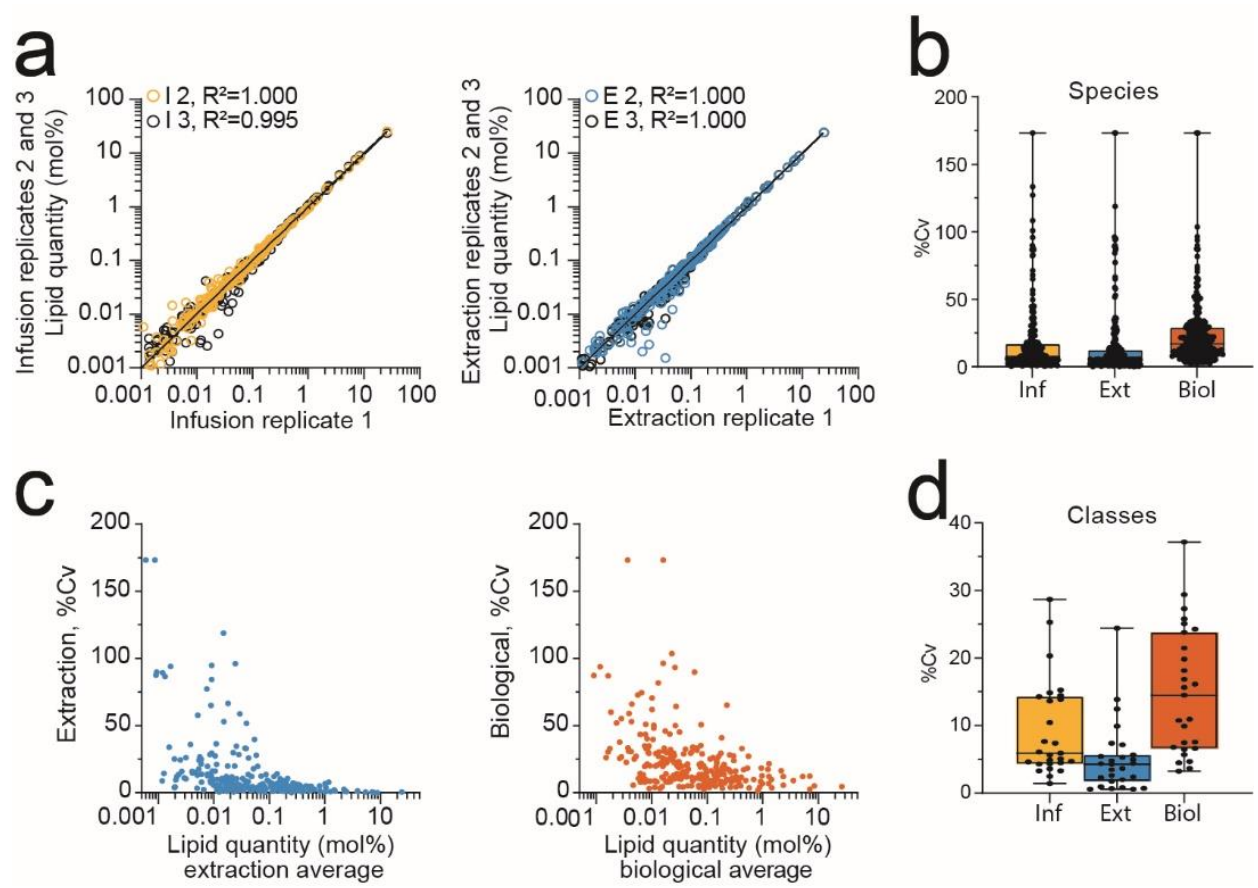

Supporting Figure 12. Reproducibility of quantitative lipidomics data acquired with our platform. (a) The determined lipid quantities of 268 lipid species (in mol\%) in one of the three analyzed infusion and extraction replicates of MEF Src cells plotted against the corresponding quantities in the other two replicates from the same replicate group. (b) Box plot of $\% \mathrm{Cv}$ values calculated for lipid species among infusion, extraction, and biological replicates. (c) $\mathrm{The}_{\mathrm{C}} \% \mathrm{Cv}_{\text {ext }}$ and $\% \mathrm{Cv}_{\text {biol }}$ values determined for the lipid species were plotted against their quantities. (d) Box plot of $\% \mathrm{Cv}$ values calculated for lipid classes among infusion, extraction, and biological replicates. 

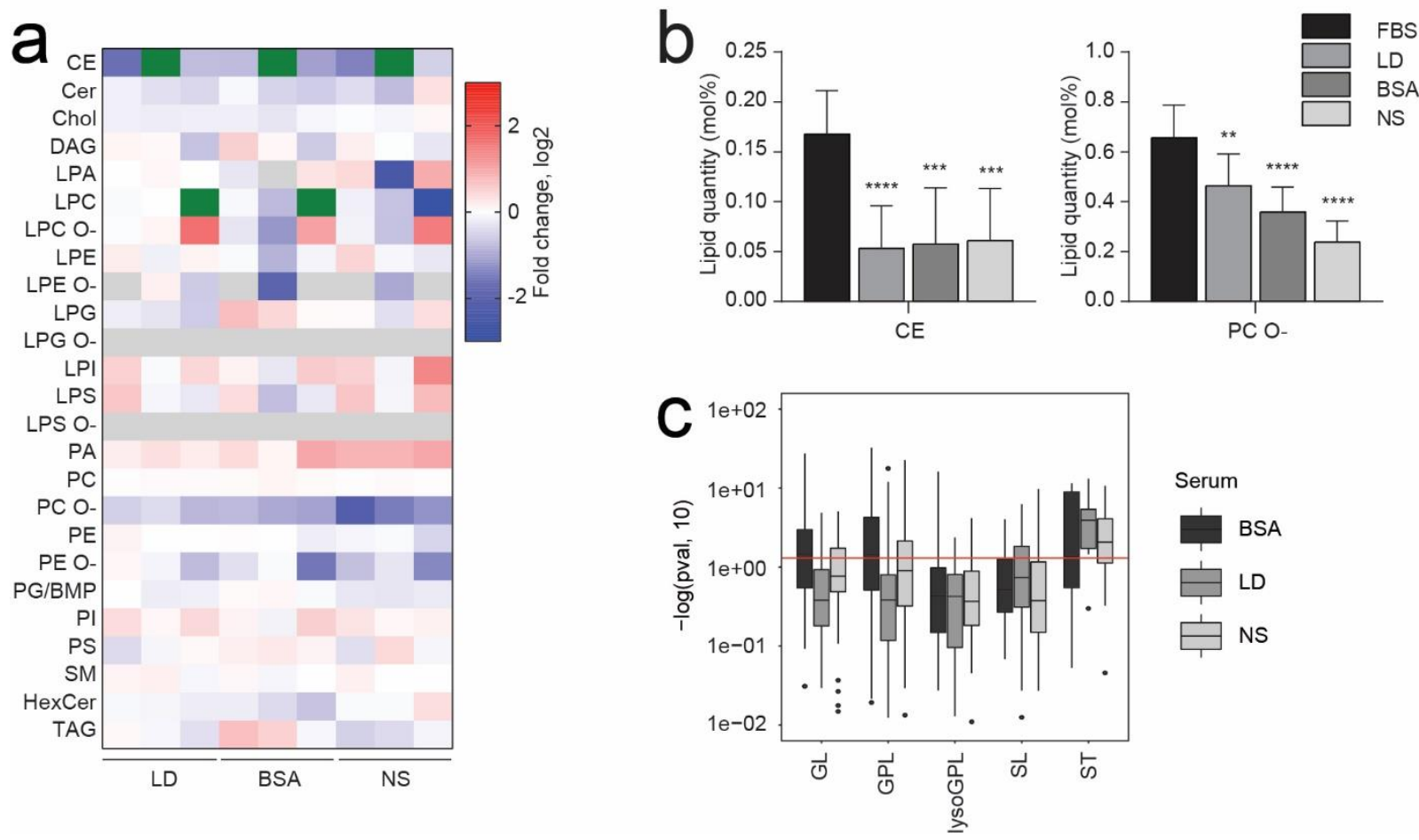

Supporting Figure 13. Effects of serum conditions on the lipidome of cultured MCF7 cells. Lipidome analysis of MCF7 cells grown for 24 hours in media containing 6\% FBS, 0.35\% (w/v) BSA, 6\% lipid-depleted FBS (LD), or with no serum (NS), $N=3$. (a) Heatmap of $\log 2$ transformed fold changes calculated from determined quantities of 25 lipid classes in cells grown under the indicated conditions compared to those in the control (FBS). (b) Bar graph of the determined quantities of CE or PC O- classes. Statistics was done with two-way ANOVA with Dunnetts' correction, $* P$-value $>0.05, * * P$-value $>0.01$, *** $P$-value $>0.001, * * * * P$-value $>0.0001$. (c) Distribution of $P$-values determined for lipid species among lipid categories. The $P$-values were obtained from linear regression modelling by comparing quantities in each condition to those in the control (FBS). Horizontal red line indicates $P$-value of 0.05 . 

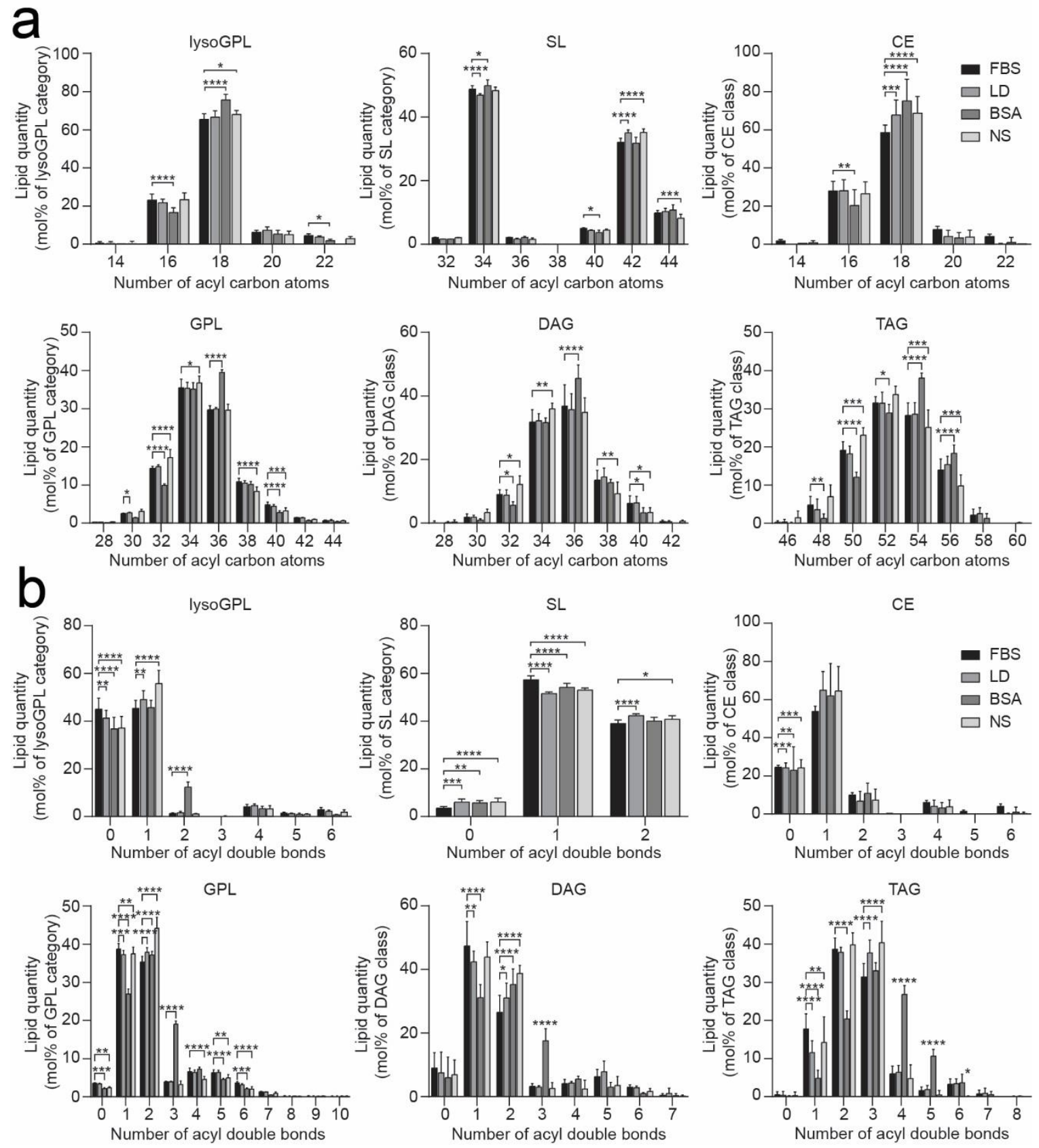

Supporting Figure 14. Effects of serum conditions on the lipidome of cultured MCF7 cells. Lipidome analysis of MCF7 cells grown for 24 hours in media containing $6 \%$ FBS, $0.35 \%$ (w/v) BSA, $6 \%$ lipid-depleted FBS (LD), or with no serum (NS), $N=3$. (a-b) Quantitative species profiles within the categories GPL, lysoGPL, and SL, and the lipid classes CE, DAG and TAG, grouping lipid species according to the number of carbon atoms (a) or the number of double bonds (b) in the acyl chain(s).The total quantities of lipid species in each groups were expressed relative to the total amount of lipids quantified within the category or class. Statistics was done with two-way ANOVA with Dunnetts' correction, $* P$-value $>0.05, * * P$-value $>0.01, * * * P$-value $>0.001, * * * * P$-value $>0.0001$. 

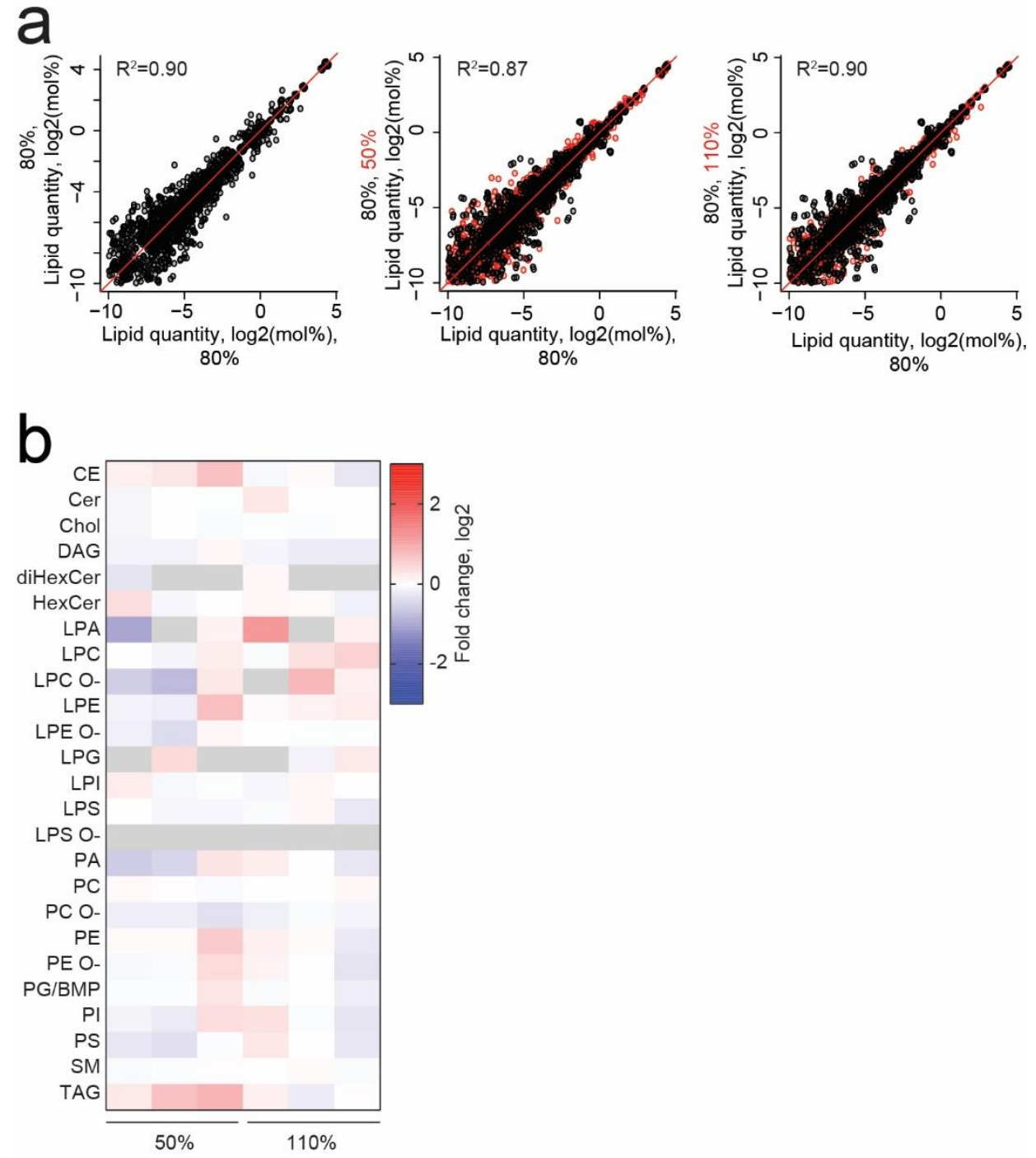

Supporting Figure 15. Effects of confluency on the lipidome of cultured HeLa cells. Lipidome analysis of HeLa cells grown for 24 hours to reach the confluences of 50\%, 80\%, or $110 \%$ (over confluency), $\mathrm{n}=3$. (a) Quantities of lipid species $(\log 2$ transformed, mol\%) determined for cells grown to the different confluence were plotted against the corresponding quantities in the control (80\% confluence). (b) Heatmap of log2 transformed fold changes calculated from determined quantities of 25 lipid classes in cells grown to the indicated confluences compared to those in the control (80\% confluence). 

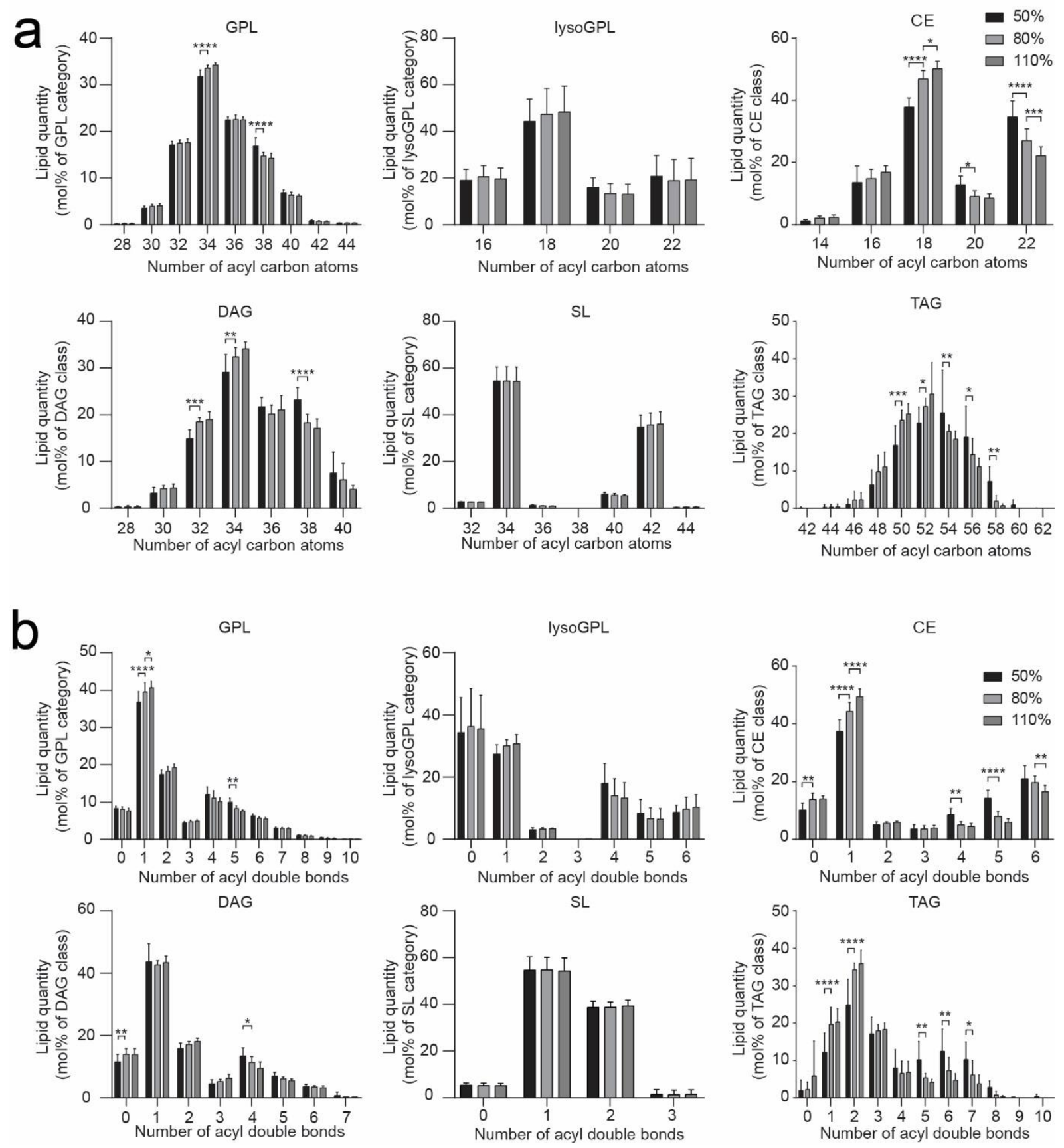

Supporting Figure 16. Effects of confluency on the lipidome of cultured HeLa cells. Lipidome analysis of HeLa cells grown for 24 hours to reach the confluences of 50\%, 80\%, or 110\% (over confluency), $\mathrm{n}=3$. (a, b) Quantitative species profiles within the categories of GPL, lysoGPL and SL and the lipid classes DAG, CE and TAG, grouping lipid species according to the number of carbon atoms (a) or the number of double bonds (b) in the acyl chain(s). The total quantities of lipid species in each groups were expressed relative to the total amount of lipids quantified within the categories and classes. Statistics was done with two-way ANOVA with Dunnetts' correction, $* P$-value $>0.05, * * P$-value $>0.01, * * *$ $P$-value $>0.001, * * * * P$-value $>0.0001$. 

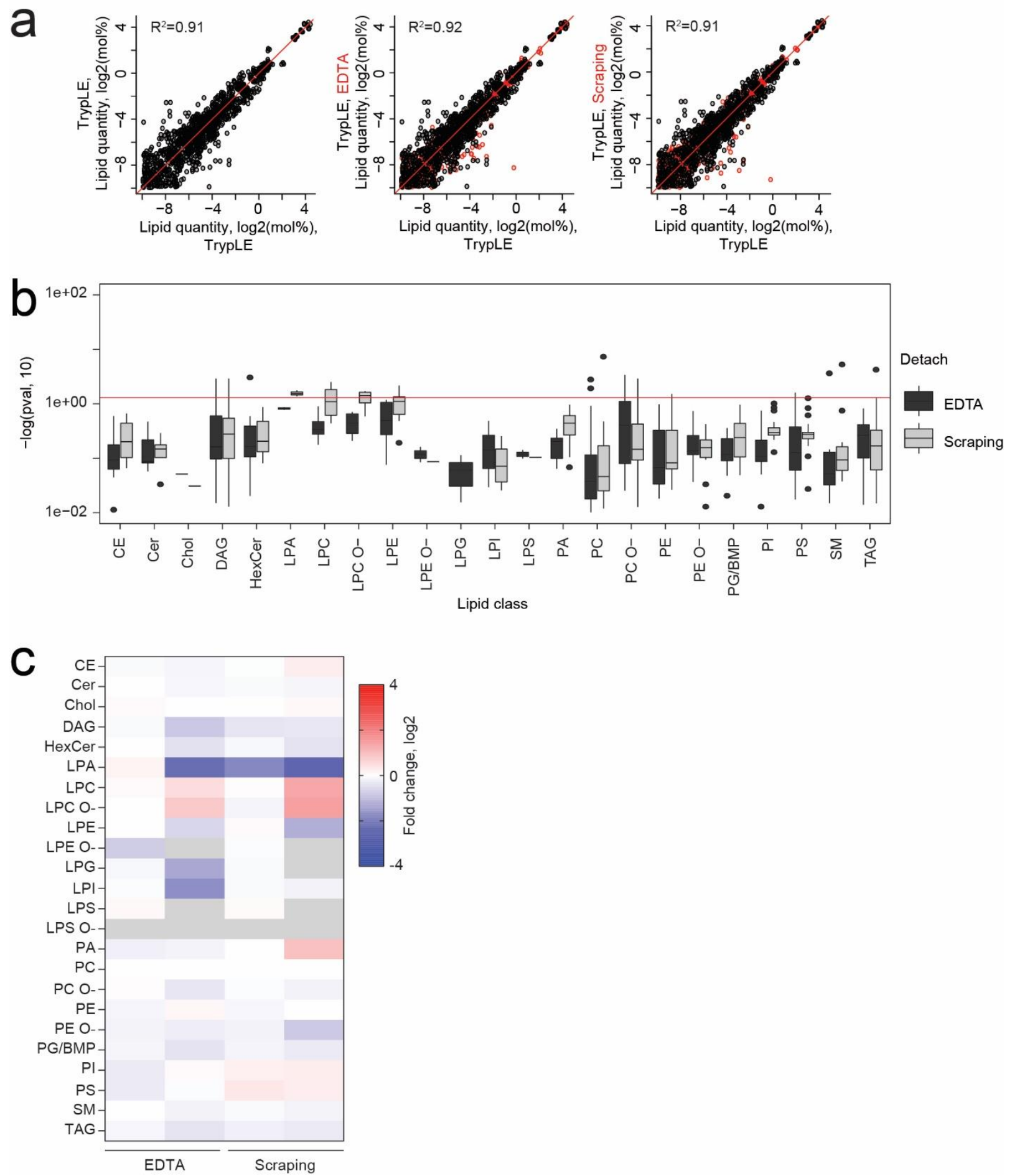

Supporting Figure 17. Effects of detachment methods on the lipidome of cultured MCF7 cells. Lipidome analysis of MCF7 cells harvested using TrypLE, EDTA, or scraping. (a) Quantities of lipid species ( $\log 2$ transformed, mol\%) determined for cells harvested by different methods were plotted against the corresponding quantities in the control (TrypLE). (b) Distribution of $P$-values determined for lipid species among lipid classes. The $P$-values were obtained from linear regression modelling by comparing quantities in each condition to those in the control (TrypLE). Horizontal red 
line indicates a $P$-value of 0.05 . (c) Heatmap of $\log 2$ transformed fold changes calculated from determined quantities of 24 lipid classes in cells detached with the indicated method compared to those in the control (TrypLE).. Statistics was done with two-way ANOVA with Dunnetts' correction, $* P$-value $>0.05$, ** $P$-value $>0.01, * * * P$-value $>0.001, * * * *$ $P$-value $>0.0001$. 

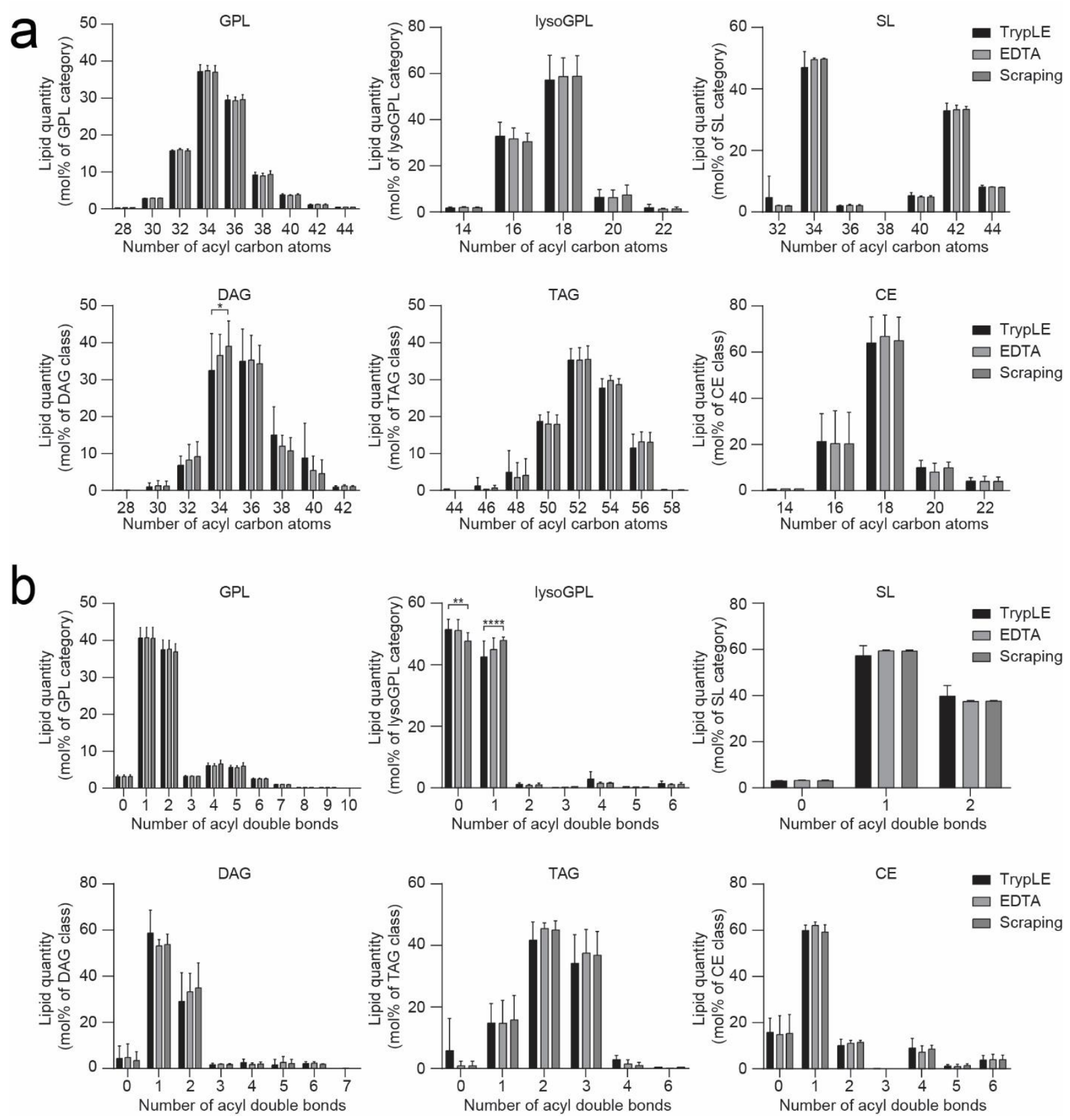

Supporting Figure 18. Effects of detachment methods on the lipidome of cultured MCF7 cells. Lipidome analysis of MCF7 cells harvested using TrypLE, EDTA, or scraping. (a, b) Quantitative species profiles within the categories of GPL, lysoGPL, and SL, and the lipid classes DAG, TAG, and CE, grouping lipid species according to the number of carbon atoms (a) or the number of double bonds (b) in the acyl chain(s). Statistics was done with two-way ANOVA with Dunnetts' correction, * $P$-value $>0.05, * * P$-value $>0.01, * * * P$-value $>0.001, * * * * P$-value $>0.0001$. 

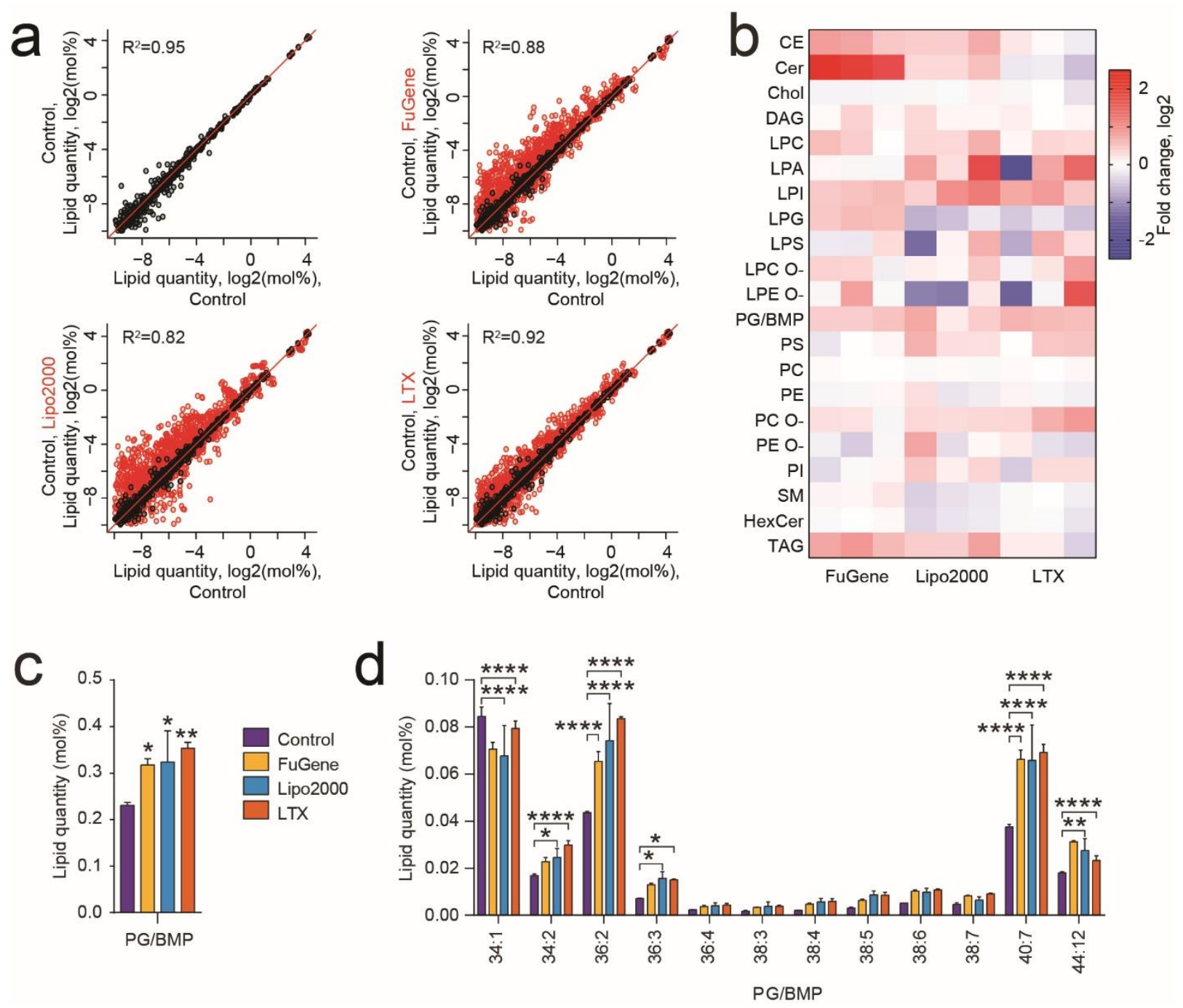

Supporting Figure 19. Effect of transfection reagents on the lipidome of cultured MCF7 cells. Lipidome analysis of MCF7 cells 24 hours after transfection with FuGene, Lipofectamine LTX, or Lipofectamine 2000, $n=2$. (a) Quantities of lipid species (log2 transformed, mol\%) determined for transfected cells were plotted against the corresponding quantities in the untransfected control (b) Heatmap of $\log 2$ transformed fold changes calculated from determined quantities of 21 lipid classes in cells transfected with the indicated reagent compared to those in the control. (c, d) Bar graphs for the determined quantities of PG/BMP class (b) and species (c). Statistics was done with two-way ANOVA with Dunnetts' correction, * $P$-value $>0.05, * * P$-value $>0.01, * * * P$-value $>0.001, * * * * P$-value $>0.0001$. 

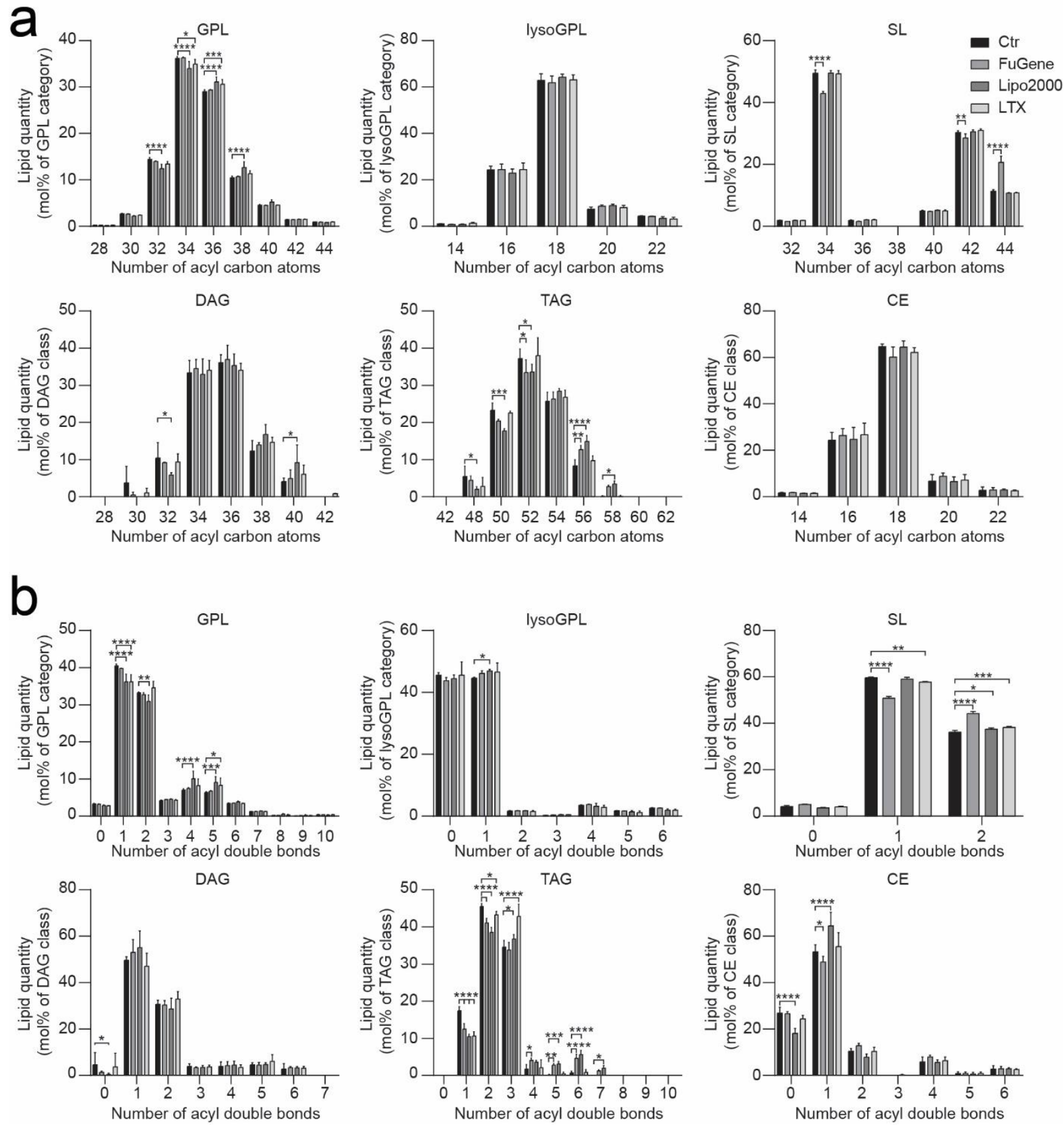

Supporting Figure 20. Effects of transfection reagents on the lipidome of cultured MCF7 cells. Lipidome analysis of MCF7 cells 24 hours after transfection with FuGene, Lipofectamine LTX, or Lipofectamine 2000, n = 2. (a, b) Quantitative species profiles within the categories of GPL, lysoGPL, and SL, and the lipid classes DAG, TAG, and CE, grouping lipid species according to the number of carbon atoms (a) or the number of double bonds (b) in the acyl chain(s). Statistics was done with two-way ANOVA with Dunnetts' correction, ${ }^{*} P$-value $>0.05, * * P$-value $>0.01, * * * P$-value $>0.001, * * * * P$-value $>0.0001$ 

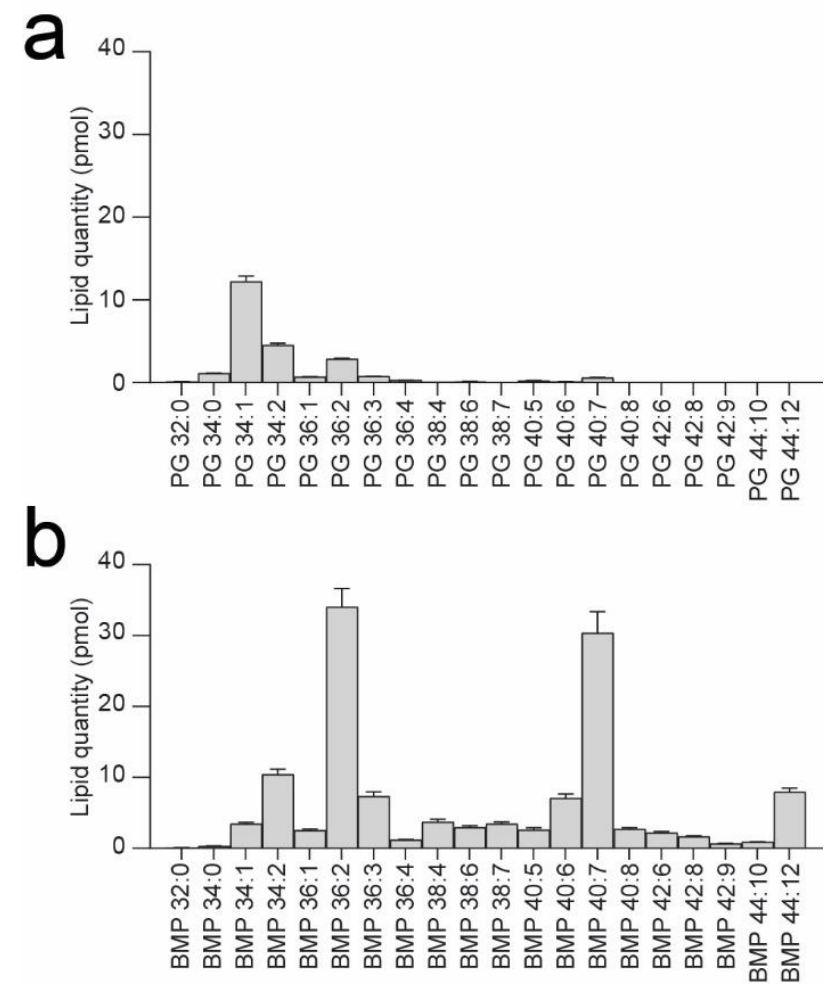

Supporting Figure 21. Quantified levels of BMP and PG species in $1 \times 10^{5}$ MCF7 cells based on LC-MS data. The determined levels of PG species (a) and BMP species (b), $\mathrm{n}=3$. 